\title{
Isomonodromic quantization of dimensionally reduced gravity
}

\author{
D. Korotkin ${ }^{1}$, H. Nicolai \\ II Institute for Theoretical Physics, Hamburg University, Luruper Chaussee 149, Hamburg 22761, Germany
}

Received 23 February 1996; revised 10 June 1996; accepted 10 June 1996

\begin{abstract}
We present a detailed account of the isomonodromic quantization of dimensionally reduced Einstein gravity with two commuting Killing vectors. This theory constitutes an integrable "midisuperspace" version of quantum gravity with infinitely many interacting physical degrees of freedom. The canonical treatment is based on the complete separation of variables in the isomonodromic sectors of the model. The Wheeler-DeWitt and diffeomorphism constraints are thereby reduced to the Knizhnik-Zamolodchikov equations for $S L(2, \mathbb{R})$. The physical states are manifestly invariant under the full diffeomorphism group. An infinite set of independent observables à la Dirac exists both at the classical and the quantum level. Using the discrete unitary representations of $S L(2, \mathbb{R})$, we construct explicit quantum states. However, the problem of satisfying the additional constraints associated with the coset space $S L(2, \mathbb{R}) / S O(2)$ remains open. We briefly discuss the possible implications of our results for string theory.
\end{abstract}

PACS: $04.60 .-\mathrm{m}$

Keywords: Quantum gravity; Integrable systems; Isomonodromic quantization; Dimensional reduction

\section{Introduction}

The purpose of this article is to explain in detail the new Hamiltonian formulation of dimensionally reduced gravity presented in [1] and to study its exact quantization as an integrable model of quantum gravity with infinitely many physical degrees of freedom on the basis of the methods introduced in [2]. Our results generalize to the more general models that one would obtain by dimensional reduction of certain matter coupled models of gravity and supergravity, but we will in this paper deal only with the stationary

\footnotetext{
${ }^{1}$ On leave of absence from Steklov Mathematical Institute, Fontanka 27, 191011 St. Petersburg, Russia.
} 
axisymmetric reduction of Einstein's theory, deferring the discussion of the Lorentzian signature (colliding plane wave) case as well as of arbitrary gravitationally coupled $\sigma$-models and the extension to locally supersymmetric models to another publication. This we do mainly in order to keep the technical complexity of the construction to a minimum and to bring out the salient features as clearly as possible.

As is well known, our general understanding of the mathematical and conceptual problems of quantum gravity is severely hampered by the scarcity of "realistic" exact solutions of the Wheeler-DeWitt (WDW) equation (for introductory reviews of the subject with many further references, see [3-5]). The known examples of exactly solvable models include pure gravity [6] (see also [7]) and supergravity [8] in three dimensions, as well as certain mini-superspace models such as static spherically symmetric gravity [9] or supersymmetric models of the type considered in [10] and references therein. Since these models describe only finitely many physical degrees of freedom, it would be desirable to find models with infinitely many physical degrees of freedom. An example of such a model is the quantum theory of cylindrical gravitational waves studied in $[11,12]$ which corresponds to a truncation of dimensionally reduced gravity for which the Ernst potential is real and (2.4) below can be transformed into a free wave equation.

Our main intention here is to demonstrate that models with infinitely many selfinteracting physical degrees of freedom can be treated exactly, and that the methods which have been developed over many years in the context of flat space integrable systems $[13,14]$ can be transplanted to quantum gravity, yielding a class of completely integrable "midi-superspace" models (which reduces to the Euclidean version of cylindrical gravitational waves for abelian groups). With regard to the conceptual problems of quantum gravity we shall adopt the pragmatic attitude that knowledge of sufficiently complicated exact solutions of the type constructed here may furnish new and essential insights also with regard to the proper interpretation of the "wave function of the universe". Indeed, notwithstanding the remaining technical difficulties, certain generic problems of quantum gravity are neatly resolved in our model. First of all, there is a well-defined Hilbert space for each isomonodromic sector; although details remain to be worked out when $N$ becomes infinite or even continuous, it is clear at least in principle how to construct the full Hilbert space as an inductive limit. Secondly, the fact that the WDW equation and diffeomorphism constraint express the invariance of the quantum state with respect to the full set of $2 \mathrm{~d}$ coordinate transformations is completely manifest; the scalar product which naturally exists in the isomonodromic subspaces is positive definite for unitary representations and respects the full diffeomorphism invariance upon restriction to the subspace of physical states. While the construction of observables à la Dirac remains a largely unsolved problem of canonical gravity in general [4], it turns out that our model admits an infinite number of independent ones, namely the monodromies associated with the singularities of the logarithmic derivative of $\Psi$ in the spectral parameter plane: these are the conserved "nonlocal charges" of matter coupled quantum gravity. The correlators of observables - the only meaningful expectation values in quantum gravity - are well defined and can be computed at least in principle. 
Our treatment of axisymmetric stationary quantum gravity relies in an essential way on the novel canonical formulation of the Ernst equation proposed in [1] and is based on the complete separation of the equations of motion and the use of the logarithmic derivative of the related $\Psi$-function with respect to the spectral parameter as the fundamental canonical variable. In [1] we have proved that; in the classical theory, the conformal factor is essentially the $\tau$-function associated with the Ernst equation. Furthermore, as shown in [2], the WDW equation for this class of models can be reduced to the Knizhnik-Zamolodchikov (KZ) equations [15]. While only the gravitationally coupled principal chiral $S U(2)$ model was analyzed from this point of view in [2], we will here extend these considerations to coset spaces and noncompact groups. Completely explicit exact solutions of the WDW equation based on the discrete unitary representations of $S U(2)$ and $S L(2, \mathbb{R})$, respectively, are thereby obtained. Unfortunately, we are not able so far to solve the additional coset constraints for the noncompact space $S L(2, \mathbb{R}) / S O(2)$ with the discrete unitary representations of $S L(2, \mathbb{R})$; rather, it appears that one will have to make use of the principal continuous series representations of $\operatorname{SL}(2, \mathbb{R})$, for which no solutions of the $\mathrm{KZ}$ equations are known so far. This is the major open problem left by the present work.

We also hope that these results may eventually enable us to address some other unsolved problems of current research in the framework of exactly solvable models. Our methods can be generalized without difficulty of principle to matter coupled gravity and supergravity because matter and gravity are unified in the group theoretical construction (for instance, to quantize Maxwell-Einstein gravity one simply would have to replace the coset space $S L(2, \mathbb{R}) / S O(2)$ by $S U(2,1) / U(2))$. Since the exact WDW functionals are built on classical solutions, we can in principle obtain solutions of the WDW equation which in a very precise sense are "close" to a given classical solution of Einstein's equations and study their $\hbar \rightarrow 0$ limits. Understanding the semiclassical limit is also a necessary prerequisite for explaining the UV divergences that would appear in a conventional perturbative treatment and that are invisible in the isomonodromic sectors which are "far away" from the perturbative regime. An intriguing aspect of our work is the possible relevance of quantum groups suggested by the link with the $\mathrm{KZ}$ equations; it appears that the notion of quantum space-time [16] may emerge quite naturally in the present framework. Our results might also shed some light on the information loss paradox for Hawking radiation: in view of persisting disagreements [17], one cannot help feeling that the Gordian knot can only be cut by finding an exactly solvable model for it.

In Section 2 we briefly recall the origin of our model as Kaluza-Klein-like dimensional reduction of $4 d$ Einstein equations with two commuting Killing vectors. Section 3 gives a classical treatment of the model in the framework of the inverse scattering method. In the isomonodromic sector of the model we rewrite the equations of motion as a system of Schlesinger-like deformation equations by introducing new canonical variables, and present the two-time Hamiltonian formulation suitable for quantization. In Section 4 we explicitly quantize this Poisson structure. The link between the WDW equations and the $\mathrm{KZ}$ equations for $S U(2)$ and $S L(2, \mathbb{R})$ is established. For the stan- 
dard unitary representations of $S U(2)$ and the unitary discrete series representations of $S L(2, \mathbb{R})$ this allows us to write down the exact WDW functional in terms of the integral representations for solutions of the $\mathrm{KZ}$ equations and to define the quantum $\tau$-function. We explain the constraints that must be satisfied when one passes from the group space $S L(2, \mathbb{R})$ to the coset space $S L(2, \mathbb{R}) / S O(2)$, which put in evidence the need for continuous (principal series) unitary representations of $S L(2, \mathbb{R})$. Since many of our results have a decidedly stringy flavor, we elaborate a little on the hints pointing towards the existence of a new kind of dual model in a separate Section 5 . In the appendix we summarize the results about unitary representations of $S L(2, \mathbb{R})$ needed in the main text.

\section{Axisymmetric stationary gravity as a nonlinear $\sigma$-model}

We will proceed from the standard metric of a stationary axisymmetric $4 d$ space-time,

$$
d s^{2}=f^{-1}\left[e^{2 k}\left(d x^{2}+d \rho^{2}\right)+\rho^{2} d \varphi^{2}\right]-f(d t+F d \varphi)^{2} .
$$

Here $(x, \rho)$ are canonical Weyl coordinates with $\rho \geq 0$ and $x \in \mathbb{R}$, and $t$ and $\varphi$ are time and angular coordinates, respectively. By assumption, the functions appearing in (2.1) depend only on $(x, p)$, and the coordinates $t$ and $\varphi$ thus play no role in the remainder. The metric coefficients appearing in (2.1) are commonly expressed in terms of the so-called Ernst potential $\mathcal{E}[18,19]$ as follows:

$$
k_{\xi}=2 i \rho \frac{\mathcal{E}_{\xi} \overline{\mathcal{E}}_{\xi}}{(\mathcal{E}+\overline{\mathcal{E}})^{2}}, \quad F_{\xi}=2 \rho \frac{(\mathcal{E}-\overline{\mathcal{E}})_{\xi}}{(\mathcal{E}+\overline{\mathcal{E}})^{2}}, \quad f=\operatorname{Re} \mathcal{E},
$$

where $\xi:=x+i \rho, \bar{\xi}:=x-i \rho$, and subscripts stand for partial derivatives throughout this paper. Next we define the symmetric matrix

$$
g=\frac{1}{\mathcal{E}+\overline{\mathcal{E}}}\left(\begin{array}{cc}
2 & i(\mathcal{E}-\overline{\mathcal{E}}) \\
i(\mathcal{E}-\overline{\mathcal{E}}) & 2 \mathcal{E} \overline{\mathcal{E}}
\end{array}\right)
$$

which can be viewed as an element of the coset space $S L(2, \mathbb{R}) / S O(2)$. Einstein's equations then imply the Ernst equation [19]

$$
\left((\xi-\bar{\xi}) g_{\xi} g^{-1}\right)_{\bar{\xi}}+\left((\xi-\bar{\xi}) g_{\bar{\xi}} g^{-1}\right)_{\xi}=0,
$$

which in this form closely resembles the equation that one would obtain for a principal chiral model. Further, they give rise to the following first order equations for the conformal factor $h \equiv e^{2 k}$ :

$$
2 k_{\xi}=(\log h)_{\xi}=\frac{\xi-\bar{\xi}}{4} \operatorname{tr}\left(g_{\xi} g^{-1}\right)^{2}, \quad 2 k_{\bar{\xi}}=(\log h)_{\bar{\xi}}=\frac{\bar{\xi}-\xi}{4} \operatorname{tr}\left(g_{\bar{\xi}} g^{-1}\right)^{2} .
$$

We will find it convenient to reexpress these equations by means of the one-form $\omega$ defined by 


$$
\omega=\frac{\xi-\bar{\xi}}{4} \operatorname{tr}\left(g_{\xi} g^{-1}\right)^{2} d \xi+\frac{\bar{\xi}-\xi}{4} \operatorname{tr}\left(g_{\xi} g^{-1}\right)^{2} d \bar{\xi} .
$$

Then (2.5) simply becomes

$$
d k=\frac{1}{2} \omega .
$$

Both (2.5) and (2.7) are consistently defined because (2.4) implies $d \omega=0$. We note already here that Eqs. (2.5) will turn into (linear combinations of) the WDW equation and the diffeomorphism constraint upon quantization.

The above equations of motion, which were obtained by dimensional reduction of the Einstein equations, can be alternatively derived directly from a $S L(2, \mathbb{R}) / S O(2)$ coset space $\sigma$-model in two space-time dimensions coupled to $2 \mathrm{~d}$ gravity and a dilaton field $\rho$ [20]. If the Euclidean worldsheet is locally parametrized by the complex coordinates $(z, \bar{z})$, the metric has the following form in the conformal gauge:

$$
d s^{2}=h(z, \bar{z}) d z d \bar{z}, \quad h \equiv e^{2 k} .
$$

A suitable Lagrangian is

$$
\mathcal{L}=\rho\left(h R+\operatorname{tr}\left(g_{z} g^{-1} g_{z} g^{-1}\right)\right),
$$

where $R$ is the Gaussian curvature of the worldsheet, i.e.,

$$
R=(\log h)_{z \bar{z}} / h,
$$

and the trace in (2.9) is appropriately normalized. As is well known from string theory, the first order equations (2.5) can be obtained from (2.9) by variation with respect to the off-diagonal elements of the worldsheet metric, so the conformal gauge condition must be temporarily relaxed. It is then obvious that these equations are completely analogous to the Virasoro conditions of string theory.

In the Lagrangian (2.9) the dilaton $\rho$ appears as an independent field, not as a coordinate. To establish the relation with the previous formulation for the metric (2.1) in terms of Weyl canonical coordinates, we note that the equation of motion for $\rho$ following from (2.9),

$$
\rho_{z z}=0,
$$

is solved by

$$
\rho(z, \bar{z})=\operatorname{Im} \xi(z),
$$

where $\xi(z)$ is a (locally) holomorphic function, and that the conformal gauge (2.8) is left intact by holomorphic reparametrizations of the complex coordinate $z$ (for which the metric remains diagonal and $h(z, \bar{z})$ is simply multiplied by a factor). Hence we can further specialize the gauge by identifying $\rho$ with one of the worldsheet coordinates as in (2.1) (global aspects of this change of variables were discussed in [21] but will not concern us here). From the $2 \mathrm{~d}$ point of view, the gravitational field $h$ and 
the matter field $g$ are coupled through the dilaton $\rho$; furthermore, the obvious solution $\rho=$ const. of (2.10) would imply $g_{\xi}=g_{\bar{\xi}}=h_{\xi}=h_{\bar{\xi}}=0$, i.e., the trivial solution for the matter fields, in which case the gravitational sector would become purely topological (strictly speaking, this argument relies on the positive definiteness of the Cartan Killing metric on the coset $S L(2, \mathbb{R}) / S O(2))$. Therefore, the model possesses no nontrivial flat space limit since the matter fields act as sources for $2 \mathrm{~d}$ gravity and thus distort the two-dimensional background geometry.

Although we do not know whether a "Wick rotation" can be rigorously justified in the context of (quantum) gravity (see, however, [22] for a recent discussion), we will occasionally take the liberty to refer to $\rho$ and $x$ as "time" and "space" coordinates, respectively, especially in connection with the canonical treatment.

\section{Classical treatment}

\subsection{Linear system and isomonodromy}

The results of this and the following section apply to solutions $g(\xi, \bar{\xi})$ of $(2.4)$ belonging to the complex general linear group $G L(n, \mathbb{C})$. The extra conditions needed to ensure that $g(\xi, \bar{\xi})$ is an element of the coset space $G / H$ and that $h(\xi, \bar{\xi}) \in \mathbb{R}$ (as required by dimensionally reduced gravity) will be presented in detail in Section 3.3.

Eq. (2.4) is the compatibility condition of the following linear system $[23,24]$ :

$$
\frac{d \Psi}{d \xi}=\frac{g_{\xi} g^{-1}}{1-\gamma} \Psi, \quad \frac{d \Psi}{d \bar{\xi}}=\frac{g_{\xi} g^{-1}}{1+\gamma} \Psi,
$$

where $\Psi(\gamma ; \xi, \bar{\xi})$ is a two-by-two matrix from which the metric (2.1) can be reconstructed. The function $\gamma(\xi, \bar{\xi})$ is a "variable spectral parameter" subject to the following (compatible) first order equations:

$$
\gamma_{\xi}=\frac{\gamma}{\xi-\bar{\xi}} \frac{1+\gamma}{1-\gamma}, \quad \gamma_{\bar{\xi}}=\frac{\gamma}{\bar{\xi}-\xi} \frac{1-\gamma}{1+\gamma} .
$$

They are solved by

$$
\gamma_{ \pm}(w ; \xi, \bar{\xi})=\frac{2}{\xi-\bar{\xi}}\left\{w-\frac{\xi+\bar{\xi}}{2} \pm \sqrt{(w-\xi)(w-\bar{\xi})}\right\}=\frac{1}{\gamma_{\mp}(w ; \xi, \bar{\xi})},
$$

with $w \in \mathbb{C}$ a constant of integration, which can be regarded as the "hidden" constant spectral parameter. In the sequel we will usually suppress the index \pm and simply write $\gamma(w ; \xi, \bar{\xi}) \equiv \gamma_{+}(w ; \xi, \bar{\xi})$. The relation $(3.3)$ can be inverted to give

$$
w=\frac{1}{4}(\xi-\bar{\xi})\left(\gamma+\frac{1}{\gamma}\right)+\frac{1}{2}(\xi+\bar{\xi}),
$$

which shows that, in our conventions, $\gamma$ is purely imaginary for real $w$. For the linear system (3.1) we can use either $\gamma$ or $w$; when $\gamma$ is expressed as a function of $w$ 
according to (3.3), the linear system (3.1) lives on the two-sheeted Riemann surface of the function $\sqrt{(w-\xi)(w-\bar{\xi})}$. Furthermore,

$$
\frac{d}{d \xi} \equiv \frac{\partial}{\partial \xi}+\frac{\gamma}{\xi-\bar{\xi}} \frac{1+\gamma}{1-\gamma} \frac{\partial}{\partial \gamma}, \quad \frac{d}{d \bar{\xi}} \equiv \frac{\partial}{\partial \bar{\xi}}+\frac{\gamma}{\bar{\xi}-\xi} \frac{1-\gamma}{1+\gamma} \frac{\partial}{\partial \gamma} .
$$

From (3.1) we immediately obtain (see also [25]) Lemma 1.

Lemma 1. The following relations hold:

$$
g_{\xi} g^{-1}=\left.\frac{2}{\xi-\bar{\xi}} \Psi_{\gamma} \Psi^{-1}\right|_{\gamma=1}, \quad g_{\bar{\xi}} g^{-1}=\left.\frac{2}{\xi-\bar{\xi}} \Psi_{\gamma} \Psi^{-1}\right|_{\gamma=-1},
$$

where the subscript $\gamma$ denotes partial differentiation with respect to $\gamma$.

Next we consider the behavior of $(d \Psi / d \xi) \Psi^{-1}$ and $(d \Psi / d \bar{\xi}) \Psi^{-1}$ in the complex $\gamma$-plane. The following theorem is a standard consequence of the formulation of classical integrable systems as a Riemann-Hilbert problem.

Theorem 1. Let the two-by-two matrix $\Psi(\gamma ; \xi, \bar{\xi})$ be subject to the following conditions:

(1) As a function of $\gamma$ the matrix $\Psi$ is holomorphic and invertible everywhere on some cover of the complex $\gamma$-plane with the exception of the points mentioned below.

(2) $\Psi$ has regular singularities at the branch points $\gamma_{j}(\xi, \bar{\xi}):=\gamma\left(w_{j} ; \xi, \bar{\xi}\right)$ for $j=$ $1, \ldots, N$ with constants $w_{j} \in \mathbb{C}$, in the vicinity of which it behaves as

$$
\Psi(\gamma ; \xi, \bar{\xi})=G_{j}(\xi, \bar{\xi}) \Psi_{j}(\gamma ; \xi, \bar{\xi})\left(\gamma-\gamma_{j}\right)^{T_{j}} C_{j} \quad \text { as } \gamma \sim \gamma_{j},
$$

where, for $\gamma \sim \gamma_{j}, \Psi_{j}(\gamma ; \xi, \bar{\xi})=\mathbf{1}+O\left(\gamma-\gamma_{j}\right)$ is holomorphic and invertible. The matrices $C_{j}$ and $T_{j}$ are constant and invertible, and constant diagonal, respectively, while the $(\xi, \bar{\xi})$-dependent matrices $G_{j}$ are assumed to be invertible.

(3) Across certain "movable" contours $\left\{L_{j}\right\}$, which connect the singular points $\gamma_{j}$ to some arbitrarily chosen but fixed and nonsingular base point $\gamma_{0} \equiv \gamma\left(w_{0} ; \xi, \bar{\xi}\right)$ and whose dependence on $(\xi, \bar{\xi})$ is determined by $(3.3)$, the boundary values of $\Psi^{-}(\gamma)$ and $\Psi^{+}(\gamma)$ are related by

$$
\Psi^{+}(\gamma ; \xi, \bar{\xi})=\Psi^{-}(\gamma ; \xi, \bar{\xi}) M_{j}(w), \quad \gamma \in L_{j},
$$

where the invertible matrices $M_{j}$ depend only on the constant spectral parameter $w$.

(4) The following asymptotic conditions hold:

$$
\begin{aligned}
& \Psi(\gamma ; \xi, \bar{\xi})=g_{\infty}+O\left(\frac{1}{\gamma}\right) \quad \text { as } \gamma \sim \infty, \\
& \Psi(\gamma ; \xi, \bar{\xi})=g(\xi, \bar{\xi})+O(\gamma) \text { as } \gamma \sim 0,
\end{aligned}
$$

where the matrix $g_{\infty}$ is constant and invertible and the matrix $g(\xi, \bar{\xi})$ is invertible. 
Then $\Psi$ obeys the linear system $(3.1)$ and $g(\xi, \bar{\xi}) \in G L(2, \mathbb{C})$ solves $(2.4)$.

Proof. Conditions (1)-(4) imply the analyticity of $\Psi_{\xi} \Psi^{-1}$ and $\Psi_{\bar{\xi}} \Psi^{-1}$ in $\gamma$ away from $\gamma= \pm 1$. The poles at $\gamma= \pm 1$ on the r.h.s. of (3.1) are produced solely by differentiation of the spectral parameter $\gamma$ with respect to $\xi$ and $\bar{\xi}$, with residues fixed by (3.10). Since condition (3.9) provides the normalization at $\gamma=\infty$, the r.h.s. of (3.1) is completely determined.

Definition 1. A solution $g(\xi, \bar{\xi})$ of (2.4) is called isomonodromic if the associated conjugation matrices $M_{j}$ are independent of $w$ (i.e., do not vary along $L_{j}$ ).

Following [26] we will refer to the set $\left\{w_{j}, T_{j}, C_{j}, L_{j}, M_{j}(w)\right\}$ as the set of monodromy data of the function $\Psi(\gamma)$ and the associated solution $g(\xi, \bar{\xi})$ of (2.4).

The logarithmic derivative ("spectral parameter current"),

$$
A(\gamma ; \xi, \bar{\xi}) \equiv \frac{\partial \Psi}{\partial \gamma} \Psi^{-1}
$$

will play a key role in the sequel. In general, $A(\gamma)$ is not single-valued as a function of $\gamma$ for nonconstant matrices $M_{j}$. It is only for isomonodromic solutions that the singularities of $A(\gamma)$ in the $\gamma$-plane are simple poles at $\gamma_{j}$. In this case $A(\gamma)$ is a meromorphic function, and we have

$$
A(\gamma)=\sum_{j=1}^{N} \frac{A_{j}}{\gamma-\gamma_{j}}
$$

(if the summation range is not indicated explicitly, sums are understood to be taken over $j=1, \ldots, N)$. The residues at the points $\gamma=\gamma_{j}$ are easily computed from (3.7),

$$
A_{j}(\xi, \bar{\xi})=G_{j} T_{j} G_{j}^{-1} .
$$

The eigenvalues of $A_{j}$ determine the ramification number of $\Psi$ at $\gamma_{j}$; if they are all rational, the number of sheets glued at $\gamma_{j}$ of the associated Riemann surface is finite, otherwise infinite. The sum of the residue matrices,

$$
A_{\infty}:=\lim _{\gamma \rightarrow \infty}(\gamma A(\gamma))=\sum_{j=1}^{N} A_{j},
$$

governs the asymptotical behavior of $\Psi(\gamma)$ at infinity. If $\Psi(\gamma)$ is regular at $\gamma=\infty$ as in (3.9), we have

$$
A_{\infty}=0 \text {. }
$$

Then we can choose $\gamma_{0}=\infty$ as the base point in Theorem 1. We shall assume (3.15) to hold throughout most of this paper. Inserting (3.12) into (3.6), we obtain

$$
g_{\xi} g^{-1}=\frac{2}{\xi-\bar{\xi}} \sum_{j} \frac{A_{j}}{1-\gamma_{j}}, \quad g_{\bar{\xi}} g^{-1}=\frac{2}{\bar{\xi}-\xi} \sum_{j} \frac{A_{j}}{1+\gamma_{j}} .
$$


This formula shows how to reconstruct the Ernst potential, and hence the space-time metric, once the residues $A_{j}$ are known.

For isomonodromic solutions, the matrices $M_{j}(w) \equiv M_{j}$ are independent of $w$ and are called monodromy matrices of the connection $A(\gamma) d \gamma$; we have

$$
M_{j}=C_{j}^{-1} e^{2 \pi i T_{j}} C_{j}
$$

By definition, they obey

$$
\frac{d M_{j}}{d \xi}=\frac{d M_{j}}{d \bar{\xi}}=0
$$

and are thus constants of motion. To express them as as path-ordered integrals we choose the same base point $\gamma_{0}$ as in Theorem 1; then

$$
M_{j}:=\mathcal{P} \exp \oint_{l_{j}} \Psi^{-1} \Psi_{\gamma} d \gamma,
$$

where the contour $l_{j}$ starts at $\gamma_{0}$, encircles the point $\gamma_{j}(\xi, \bar{\xi})$ and returns to $\gamma_{0}$; of course, $M_{j}$ does not depend on the choice of $\gamma_{0}$ as is already obvious from (3.17). For $\gamma_{0}=\infty$ we get

$$
M_{j}=g_{\infty}^{-1}\left(\mathcal{P} \exp \oint_{l_{j}} A(\gamma) d \gamma\right) g_{\infty} .
$$

The eigenvalues of the matrices $\log M_{j}$ and $2 \pi i A_{j}$ are the same; however, the explicit relation between them is highly nonlocal. The "monodromy at infinity",

$$
\prod_{j=1}^{N} M_{j}=M_{\infty}=\exp \left(2 \pi i A_{\infty}\right),
$$

is equal to 1 as a corollary of the assumed regularity of $\Psi(\gamma)$ at $\gamma=\infty$.

An obvious question at this point concerns the status of the isomonodromic solutions among all solutions of (2.4). At first sight (3.12) looks like a strong constraint on the possible solutions, but in fact it is not. Apart from the assumed analyticity of $\Psi$, the only true assumption that goes into (3.12) is the absence of essential singularities of $\Psi$ as a function of $\gamma$ (this assumption must, however, be relaxed for locally supersymmetric theories where higher order "rigid" poles at $\gamma= \pm 1$ appear in the linear system [27]); then (3.12) is just the analog of the well-known statement that the logarithmic derivative of an analytic function can be represented as a sum over its poles. In fact, almost all known exact solutions of (2.4) are isomonodromic. So, for multisoliton solutions of Einstein's equations [24], all of the matrices $M_{j}$ are proportional to the unit matrix such that all eigenvalues of $A_{j}$ are half integer, and for the finite gap (algebro-geometric) solutions constructed in [28,29] the matrices $M_{j}$ are either anti-diagonal (i.e., with zeros on the diagonal) or again proportional to 1. For solutions expressible in terms of Painlevé transcendents [30], the matrices $M_{j}$ are triangular. The only examples of solutions which 
are not strictly isomonodromic in the sense that the sum in (3.12) is infinite and that there is an accumulation point of the poles at infinity are the periodic analogs of the axisymmetric static solutions constructed in [31,21]. Evidently, such solutions may be obtained from the $\mathrm{N}$-soliton solutions by a limiting procedure. Indeed, we can at least in principle approximate an arbitrary solution of (2.4) by isomonodromic ones (perhaps including higher order poles) if we approximate a given nonconstant function $M_{j}(w)$ by step functions. If this procedure could be justified rigorously we could claim that our present treatment also covers the general case where the sum in (3.12) is replaced by an integral. However, in spite of some work in this direction for other integrable systems [32], the results obtained so far are still inconclusive. Rigorously speaking, we are thus considering a truncation of the total phase space by assuming (3.12), and the question of whether we can get the full phase space as a union (in a suitable completion) of its isomonodromic subsectors remains open for the moment. We conjecture that a complete treatment of the model will allow us to rigorously justify the restriction to the isomonodromic sectors, which is introduced in (3.12) "by hand". Naturally this question also bears upon the precise definition of the Hilbert space of the quantum theory.

The analyticity properties of $\Psi(\gamma)$ have also been discussed extensively in [33]. To understand the difference between the approaches of [24] and [33] we note that, together with a given $\Psi(\gamma)$, the function $\Psi(\gamma) S(w)$ also solves the linear system (3.1) for any nondegenerate matrix $S(w)$. While the multiplication by such a matrix does not affect the $(\xi, \bar{\xi})$-dependence, it does alter the analyticity properties of $\Psi$ as a function of $\gamma$ (and hence the prescription for extracting the solution $g(\xi, \bar{\xi})$ from it). As a consequence, the expansion (3.12) is also modified because $\partial_{\gamma} S=\partial_{w} S \partial_{\gamma} w \neq 0$. In [33] this residual freedom in the choice of $\Psi$ is completely eliminated by demanding $\Psi(\gamma)$ to be holomorphic a certain neighborhood of the origin (e.g., the unit disk in the $\gamma$-plane), whereas no such restriction on the location of the poles in the $\gamma$-plane is made in [24]. The precise relation between these different "pictures" will be further clarified in Section 3.3 when we discuss the coset constraints.

\subsection{Deformation equations and $\tau$-function}

Substituting (3.16) into (3.1) and demanding compatibility between (3.1) and (3.12), we get [1]

$$
\frac{\partial A_{j}}{\partial \xi}=\frac{2}{\xi-\bar{\xi}} \sum_{k \neq j} \frac{\left[A_{k}, A_{j}\right]}{\left(1-\gamma_{k}\right)\left(1-\gamma_{j}\right)}, \quad \frac{\partial A_{j}}{\partial \bar{\xi}}=\frac{2}{\bar{\xi}-\xi} \sum_{k \neq j} \frac{\left[A_{k}, A_{j}\right]}{\left(1+\gamma_{k}\right)\left(1+\gamma_{j}\right)} .
$$

We repeat that these deformation equations as well as the definition of the $\tau$-function to be presented below are valid generally for the groups $G L(n, \mathbb{C})$. Eqs. (3.22) may also be represented in "Lax form", viz.

$$
\frac{\partial A_{j}}{\partial \xi}=\left[\left.U\right|_{\gamma=\gamma_{j}}, A_{j}\right], \quad \frac{\partial A_{j}}{\partial \bar{\xi}}=\left[\left.V\right|_{\gamma=\gamma_{j}}, A_{j}\right]
$$


where

$$
U=\frac{g_{\xi} g^{-1}}{1-\gamma}, \quad V=\frac{g_{\xi} g^{-1}}{1+\gamma} .
$$

This form of (3.22) is "gauge-covariant" with respect to the transformation

$$
\tilde{\Psi}=\Omega(\xi, \bar{\xi}) \Psi,
$$

in the sense that the transformed function $\tilde{\Psi}$ satisfies the linear system $d \tilde{\Psi} / d \xi=\tilde{U} \tilde{\Psi}$, $d \tilde{\Psi} / d \bar{\xi}=\tilde{V} \tilde{\Psi}$ with

$$
\tilde{U}=\Omega_{\xi} \Omega^{-1}+\Omega U \Omega^{-1}, \quad \tilde{V}=\Omega_{\bar{\xi}} \Omega^{-1}+\Omega V \Omega^{-1} .
$$

Clearly, the matrix functions $A_{j}$ transform as $A_{j} \rightarrow \tilde{A}_{j}=\Omega A_{j} \Omega^{-1}$ under (3.24). The transformed matrices $\tilde{A}_{j}$ then obey the same linear system $(3.23)$ with the pair $(U, V)$ replaced by $(\tilde{U}, \tilde{V})$.

We are now in a position to formulate Theorem 2 .

Theorem 2. Let $\left\{w_{j} \in \mathbb{C} ; j=1, \ldots, N\right\}$ be an arbitrary set of complex constants and $A_{j}=A_{j}(\xi, \bar{\xi}) \in g l(2, \mathbb{C})$. Then

(1) The two matrix differential equations (3.22) are compatible if $\gamma_{j}=\gamma\left(w_{j} ; \xi, \bar{\xi}\right)$.

(2) The linear system (3.16) for $g(\xi, \bar{\xi})$, where $\left\{A_{j}(\xi, \bar{\xi})\right\}$ is an arbitrary solution of (3.22), is also compatible, and its solution $g(\xi, \bar{\xi}) \in G L(2, \mathbb{C})$ satisfies Eq. (2.4).

Proof. The compatibility of Eqs. (3.22) can be checked by a straightforward computation. Combining (3.16) and (3.22) we recover the (complexified) Ernst equation (2.4).

Remarkably, the dependence of the (complexified) Ernst equation and its associated linear system on the variables $\xi$ and $\bar{\xi}$ has been completely decoupled by Theorem 2 . Therefore the problem of solving Einstein's equations in this reduction has been reduced to integrating a system of ordinary matrix differential equations, which are automatically compatible unlike the original linear system (3.1). All information about the degrees of freedom is thereby encoded into the "initial values", i.e., the set of matrices $A_{j}^{(0)} \equiv$ $A_{j}\left(\xi^{(0)}, \bar{\xi}^{(0)}\right)$, where $\left(\xi^{(0)}, \bar{\xi}^{(0)}\right)$ is an arbitrarily chosen base point, and the value at any other point can be consistently obtained by integration along any curve connecting it to the base point ${ }^{2}$. The matrices $A_{j}^{(0)}$ are also the appropriate phase space variables for the matter sector, as we will see in Section 3.4. Accordingly, we will regard the functions $A_{j}(\xi, \bar{\xi})$ rather than $\Psi(\gamma ; \xi, \bar{\xi})$ as the basic quantities from now on, and

\footnotetext{
${ }^{2}$ Although we are dealing with an elliptic rather than a hyperbolic partial differential equation, there is no paradox here because the "initial values" also determine the behavior at infinity via the matrix $A_{\infty} \equiv A_{\infty}^{(0)}=$ $-\sum_{j} A_{j}^{(0)}$ (which is a constant of motion by Lemma 2 below) as appropriate for an elliptic boundary value problem.
} 
relate the system (3.22) directly to the (complexified) Ernst equation (2.4). With this parametrization of the phase space, the isomonodromic subsectors can be treated separately, as they are stable with respect to the evolution equations for arbitrary choices of the "soliton number" $N$ and the points $w_{j}$.

As an immediate consequence of (3.22) we can construct integrals of motion, confirming the statements after (3.18).

Lemma 2. Let $\left\{A_{j}\right\}$ be an arbitrary solution of the system (3.22). Then the variables $A_{\infty} \equiv \sum_{j} A_{j}, \operatorname{tr} A_{j}$ and $\operatorname{tr} A_{j}^{2}$ (and thus all eigenvalues of the two-by-two matrices $A_{j}$ ) are $(\xi, \bar{\xi})$-independent, hence constants of motion.

Notice that the lemma is in accord with the original definition of $A_{j}$ in (3.13), but more general since we now consider arbitrary solutions of (3.22). As a corollary, we conclude that the sum

$$
\sum_{j<k} \operatorname{tr}\left(A_{j} A_{k}\right)=\frac{1}{2} \operatorname{tr} A_{\infty}^{2}-\frac{1}{2} \sum_{j} \operatorname{tr} A_{j}^{2}
$$

is likewise $(\xi, \bar{\xi})$-independent. The above constants of motion will give rise to observables in the canonical framework.

To each solution $\left\{A_{j}\right\}$ of (3.22) we can associate the following closed one-form [26]:

$$
\omega_{0}(\xi, \bar{\xi})=\sum_{j<k} \operatorname{tr}\left(A_{j} A_{k}\right) d \log \left(\gamma_{j}-\gamma_{k}\right),
$$

where the exterior derivative $d$ is to be taken with respect to the deformation parameters $(\xi, \bar{\xi})$. The closure condition $d \omega_{0}=0$ may be directly verified by use of (3.22) and (3.2). Following the general prescription given in [26], we have Definition 2.

Definition 2. The function $\tau(\xi, \bar{\xi})$ defined by

$$
d \log \tau=\omega_{0}
$$

is called the $\tau$-function of the Ernst equation.

We will now show that the $\tau$-function has a very definite physical meaning in our context: up to an explicit factor, it is just the conformal factor $h \equiv e^{2 k}$ ! To establish this result, we first substitute (3.16) into (2.6); then using (3.2) and (3.27), we obtain

$$
\begin{aligned}
\omega= & \omega_{0}+\frac{1}{\xi-\bar{\xi}} \sum_{j} \operatorname{tr} A_{j}^{2}\left\{\frac{d \xi}{\left(1-\gamma_{j}\right)^{2}}-\frac{d \bar{\xi}}{\left(1+\gamma_{j}\right)^{2}}\right\} \\
& +\sum_{j<k} \operatorname{tr}\left(A_{j} A_{k}\right) d \log (\xi-\bar{\xi}) .
\end{aligned}
$$

By (3.26) all extra terms on the r.h.s. of (3.29) may be explicitly integrated. Using (3.2), (3.29) and the relation 


$$
\begin{aligned}
& \frac{2}{\xi-\xi}\left\{\left(\frac{1}{\left(1-\gamma_{j}\right)^{2}}-\frac{1}{2}\right) d \xi-\left(\frac{1}{\left(1+\gamma_{j}\right)^{2}}-\frac{1}{2}\right) d \vec{\xi}\right\} \\
& =d\left(\log \frac{\gamma_{j}^{2}}{(\xi-\bar{\xi})\left(1-\gamma_{j}^{2}\right)}\right)=d\left(\log \frac{\partial \gamma_{j}}{\partial w_{j}}\right)
\end{aligned}
$$

we arrive at Theorem 3 .

Theorem 3. The conformal factor $h$ defined by (2.6) and (2.7), and the $\tau$-function defined by (3.28) are related by

$$
h\left(\xi, \bar{\xi} ;\left\{w_{j}\right\}\right)=C(\xi-\bar{\xi})^{\left(\operatorname{tr} A_{\infty}^{2}\right) / 2} \prod_{j}\left\{\frac{\partial \gamma_{j}}{\partial w_{j}}\right\}^{\left(\operatorname{tr} A_{j}^{2}\right) / 2} \tau\left(\xi, \bar{\xi} ;\left\{w_{j}\right\}\right)
$$

where $C$ is an integration constant.

Observe that the notation in (3.30) can be further unified by writing (see (3.4))

$$
\frac{4}{\xi-\bar{\xi}}=\left.\frac{\partial \gamma}{\partial w}\right|_{\gamma=\infty}
$$

For $A_{\infty}=0$, the first factor in (3.30) can be dropped. Also, for the group $G L(2, \mathbb{C})$, the function $h(\xi, \bar{\xi})$ is complex in general. Apart from its structural content this theorem is more general than previous results, where the conformal factor was computed only for multisoliton solutions [24]. It would be interesting to find out how it is related to the "cocycle formula" of [33].

The above formulas simplify considerably for abelian groups - corresponding to the Euclidean version of cylindrical waves for $G=G L(1, \mathbb{R})=\mathbb{R}^{+}$- or if the residue functions $A_{j}$ are in the Cartan subalgebra of the non-abelian group under consideration (which for $G=S L(2, \mathbb{R})$ would give the multi-Schwarzschild solutions). Namely, if the commutators on the r.h.s. side of (3.22) vanish, all $A_{j}$ are constant. One can then immediately write down the solution of the linear system, which reads

$$
\Psi(\gamma ; \xi, \bar{\xi})=F(\xi, \bar{\xi}) \prod_{j=1}^{N}\left(\gamma-\gamma_{j}\right)^{A_{j}}
$$

The undetermined function $F(\xi, \bar{\xi})$ is fixed by imposing the proper $\gamma$-dependence in (3.1) and given by

$$
F(\xi, \bar{\xi})=(\xi-\bar{\xi})^{\sum A_{j}}
$$

(thus $F \equiv 1$ for $A_{\infty}=0$ ). From this we can immediately read off the solution of (2.4) with $\varphi \equiv \log g$,

$$
\varphi(\xi, \bar{\xi})=\sum_{j=1}^{N} A_{j} \log \left((\xi-\bar{\xi}) \gamma\left(w_{j} ; \xi, \bar{\xi}\right)\right)
$$


(3.33) bears some similarity to the mode expansion of a string target space coordinate in terms of oscillators. However, it is better to think of (3.33) as an expansion in terms of coherent states.

\subsection{Reality conditions and coset constraints}

The solutions $g(\xi, \bar{\xi})$ obtained from the deformation equations (3.22) in general are neither symmetric nor even in $S L(2, \mathbb{R})$. We must therefore impose extra conditions in order to ensure that $g(\xi, \bar{\xi}) \in S L(2, \mathbb{R}) / S O(2)$, i.e., that $g$ is real and symmetric, and that the conformal factor is real. For this purpose we need both a reality condition on the moving poles $\gamma_{j}$ (and thus on the parameters $w_{j}$ ) as well as certain extra constraints on $\Psi$, the monodromy matrices or the $\tau$-function. These conditions may be formulated in several equivalent ways which we shall now present.

Quite generally it is clear that for $g$ to be in a given Lie group, the residues $A_{j}$ in (3.12) must be elements of the associated (complexified) Lie algebra; consequently, for simple Lie groups, we have $\operatorname{tr} A_{j}=0$. Depending on which real form one is dealing with, the parameters $w_{j}$ and the matrices $A_{j}$ are subject to certain reality conditions. In the case at hand, these follow from the simple requirement that the two expressions in (3.16) are complex conjugate to one another for real $\mathrm{g}$. It is important here that only the sums on the r.h.s. of (3.16) are constrained in this way, so that the individual matrices $A_{j}$ can (and will!) still belong to the complexified Lie algebra $s l(2, \mathbb{C}$ ).

The conditions needed to make $g$ an element of a coset space rather than a group are more subtle and require some explanation. An essential observation at this point is that, for symmetric $g(\xi, \vec{\xi})$, one can prove from (3.1) that the matrix

$$
\mathcal{M}(\gamma ; \xi, \bar{\xi}):=\Psi^{\mathrm{t}}\left(\frac{1}{\gamma} ; \xi, \bar{\xi}\right) g^{-1}(\xi, \bar{\xi}) \Psi(\gamma ; \xi, \bar{\xi})
$$

is annihilated by the operators (3.5),

$$
\frac{d \mathcal{M}}{d \xi}=\frac{d \mathcal{M}}{d \bar{\xi}}=0
$$

and therefore depends only on the constant spectral parameter, i.e.,

$$
\mathcal{M}(\gamma(w ; \xi, \bar{\xi}) ; \xi, \bar{\xi}) \equiv \mathcal{M}(w)
$$

(by a slight abuse of notation, we write $\mathcal{M}$ on the r.h.s., too). From the invariance of $w$, and hence of $\mathcal{M}(w)$, under the involution $\gamma \rightarrow \gamma^{-1}$ we immediately obtain

$$
\mathcal{M}^{\mathrm{t}}(w)=\mathcal{M}(w)
$$

The constancy of the matrix $\mathcal{M}$ (as a function of the coordinates) was already noticed in [24]; in [33] it played an important role in reaching a systematic understanding of axisymmetric stationary solutions of Einstein's equations. $\mathcal{M}(w)$ is called "monodromy matrix" in [33], but obviously it must not be confused with the matrices $M_{j}$ defined in (3.19). In both [24] and [33] the problem of finding solutions to Einstein's equations 
is reformulated as a Riemann-Hilbert problem by reducing (3.1) to the factorization problem (3.34). In the description of [33], $\mathcal{M}=1$ would correspond to the trivial vacuum solution (i.e., Minkowski space), while nontrivial solutions are characterized by a nonconstant $\mathcal{M}(w)$. By contrast, in [24] $\Psi$ is fixed by demanding $\mathcal{M}(w) \equiv \mathbf{1}$ for all solutions. These two possibilities, therefore, correspond to two different descriptions of the same solution of Einstein's equation ${ }^{3}$. It is crucial that the asymptotic expansion (3.12) is compatible with the $(\xi, \bar{\xi})$-independence of the matrix (3.34) only if $\mathcal{M}$ is $w$-independent. For this reason we will adopt the prescription of [24] rather than that of [33] in the rest of this paper (this also relieves us of the need to worry about a new name for $\mathcal{M}(w))$.

The main idea is now to turn the above statement around and to link the desired symmetry of $g$ to the constancy of (3.34).

Theorem 4. Suppose that in addition to the conditions of Theorem 1, function $\Psi$ satisfies

$$
\Psi(-\bar{\gamma})=\overline{\Psi(\gamma)}
$$

and

$$
\Psi^{\mathrm{t}}\left(\frac{1}{\gamma}\right) g^{-1} \Psi(\gamma)=g_{\infty} .
$$

Then the constants of integration in (2.7) and (2.3) may be chosen such that $g(\xi, \bar{\xi})$ is symmetric and $h(\xi, \bar{\xi}) \in \mathbb{R}$; in particular, (3.39) implies $g_{\infty} \in S L(2, \mathbb{R}) / S O(2)$.)

Proof. From (3.38) we immediately obtain

$$
\overline{A(\gamma)}=-A(-\bar{\gamma}),
$$

so we have in particular $A(1)=-A(-1)$; the reality condition $g(\xi, \bar{\xi}) \in S L(2, \mathbb{R})$ then follows from (3.6). Setting $\gamma=\infty$ in (3.39) and making use of the assumed asymptotic properties (3.9) and (3.10) of $\Psi$ we get $g=g^{\mathrm{t}}$; on the other hand, taking $\gamma=0$ we obtain $g_{\infty}^{\mathrm{t}}=g_{\infty}$.

In the form stated above, the conditions suffer from the drawback that the solution $g(\xi, \xi)$ of the Ernst equation appears explicitly in (3.39), so the coset property can only be verified a posteriori, i.e., after the solution has already been constructed. As a first step towards the elimination of $g$ we reformulate the relevant conditions in terms of the poles $\gamma_{j}$ and the matrices $A_{j}$.

Lemma 3. Theorem 4 remains valid if we require $A_{\infty}=0$ and replace conditions (3.38) and (3.39) by the invariance of the set $\left\{A_{j}, \gamma_{j}\right\}$ with respect to the involutions

\footnotetext{
${ }^{3}$ The precise formula can be worked out by factoring the "monodromy matrix" of [33] as $\mathcal{M}_{B M}(w)=S^{\mathrm{t}}(w) S(w)$. The linear system functions of [33] and [24] are then related by $\Psi_{B M}(\gamma)=$ $\Psi_{B Z}(\gamma) S(w(\gamma ; \xi, \bar{\xi})$ ). This formula also explains why multisoliton solutions correspond to rational $\Psi(\gamma)$ in [33], whereas square root branch cuts (and hence half integer eigenvalues of $A_{j}$ ) appear in the description of $[24]$.
} 


$$
\begin{aligned}
A_{j} \rightarrow \overline{A_{j}}, & \gamma_{j} \rightarrow-\bar{\gamma}_{j} \\
A_{j}^{\mathrm{t}} \rightarrow-g^{-1} A_{j} g, & \gamma_{j} \rightarrow \frac{1}{\gamma_{j}} .
\end{aligned}
$$

Proof. Clearly, the first condition is equivalent to (3.40) by (3.12) (see also (3.16)). To prove the second part of the lemma, we differentiate both sides of (3.39) with respect to $\gamma$; noticing that the r.h.s. gives zero, a little algebra leads to

$$
\frac{1}{\gamma^{2}} A^{\mathrm{t}}\left(\frac{1}{\gamma}\right)=g^{-1} A(\gamma) g,
$$

or

$$
\frac{1}{\gamma} \sum_{j=1}^{N} \frac{A_{j}^{\mathrm{t}}}{\gamma^{-1}-\gamma_{j}}=\gamma \sum_{j=1}^{N} \frac{g^{-1} A_{j} g}{\gamma-\gamma_{j}} .
$$

Now performing the substitution (3.42) on the 1.h.s., we arrive at

$$
\sum_{j=1}^{N} \gamma \frac{A_{j}}{\gamma-\gamma_{j}}=\sum_{j=1}^{N} \gamma_{j} \frac{A_{j}}{\gamma-\gamma_{j}},
$$

which is indeed fulfilled provided that $A_{\infty}=\sum A_{j}=0$.

The coset condition (3.42) can be satisfied by taking $N=2 n$ and assuming

$$
\gamma_{j+n}=\frac{1}{\gamma_{j}}, \quad A_{j+n}^{\mathrm{t}}=-g^{-1} A_{j} g .
$$

While the involution $\gamma \rightarrow \gamma^{-1}$ has only two fixed points at $\gamma= \pm 1$, the anti-involution $\gamma \rightarrow-\bar{\gamma}$ leaves all points $\gamma_{j}$ fixed for which $w_{j} \in \mathbb{R}$. Thus (3.41) implies that $n=m+2 l$ and that for $j=1, \ldots, m$ and $j=n+1, \ldots, n+m$,

$$
\gamma_{j}=-\overline{\gamma_{j}}, \quad A_{j}=\overline{A_{j}},
$$

whereas for $j=m+1, \ldots, m+l$ and $j=n+m+1, \ldots, n+m+l$,

$$
\gamma_{j}=-\overline{\gamma_{j+l}}, \quad A_{j}=\overline{A_{j+l}} .
$$

Thus for (3.47) we have $w_{j} \in \mathbb{R}$ and $A_{j} \in \operatorname{sl}(2, \mathbb{R})$, whereas for (3.48) $w_{j}$ is complex and we have $A_{j} \in \operatorname{sl}(2, \mathbb{C})$.

The complete elimination of $g(\xi, \bar{\xi})$ is achieved by reformulating the above constraints in terms of the monodromy matrices and the $\tau$-function. It is straightforward to deduce from (3.8) and (3.39) the conditions

$$
M_{j+n}^{\mathrm{t}}=g_{\infty} M_{j}^{-1} g_{\infty}^{-1}
$$

which do not contain $g(\xi, \bar{\xi})$ anymore. By the one-to-one correspondence between the solutions of (2.4) and the monodromy data [26], we therefore have Theorem 5. 
Theorem 5. Let $\Psi$ obey the reality condition (3.38) and let the monodromy matrices satisfy the relations (3.49). Furthermore suppose that the eigenvalues of each matrix $A_{j}$ do not differ by integers and $\operatorname{tr} A_{j}^{2}=\operatorname{tr} A_{j+n}^{2}$. Then the constants of integration in (3.6) and (2.7) may be chosen in such a way that $g(\xi, \bar{\xi})$ solves the Ernst equation (2.4), is real and symmetric, and the conformal factor $h$ is real.

Finally, we give the necessary condition for the fulfillment of the constraints in terms of the $\tau$-function.

Corollary 1. Suppose that the same conditions as in Lemma 3 hold. Then

$$
\tau\left(\frac{1}{\gamma_{1}}, \ldots, \frac{1}{\gamma_{N}}\right)=\tau\left(\gamma_{1}, \ldots, \gamma_{N}\right)
$$

Proof. From (3.27) it is obvious that the $\tau$-function depends only on the traces of products of the matrices $A_{j}$. Then (3.39) is an immediate consequence of the invariance under (3.42).

The symmetry and reality conditions as well as the regularity properties of $\Psi$ are preserved by the Ehlers group $S L(2, \mathbb{R})[19]$. An Ehlers transformation is characterized by a matrix $Q \in S L(2, \mathbb{R})$ and acts on $\Psi$ as

$$
\Psi \rightarrow \widetilde{\Psi}=Q^{\mathrm{t}} \Psi Q
$$

From (3.11) we infer that (3.51) induces the following transformation on the residue matrices:

$$
A_{j} \rightarrow \widetilde{A}_{j}=Q^{\mathrm{t}} A_{j} Q^{t-1} .
$$

It is known that the Ehlers group admits an infinite extension, the so-called Geroch group [34], which also acts on the space of axisymmetric stationary solutions. Save for some scattered remarks we will not consider this group here; see, however, [35,33,36] for a description of this group and its generalizations in the framework of dimensional reduction.

\section{4. "Two-time" Hamiltonian formalism}

We will now present the canonical formulation of the results described in the foregoing sections. For this purpose we adopt a "two-time" Hamiltonian formalism with the two "times" corresponding to the lightcone coordinates $\xi$ and $\bar{\xi}$. One major advantage of this procedure is that the quantum theory is manifestly covariant under $2 \mathrm{~d}$ coordinate transformations, a feature which is far from obvious (and possibly not even true) for the ADM formulation of canonical quantum gravity (see, e.g., [37] for a recent discussion). In the case at hand, the two-time Hamiltonian structure giving rise to the equations of motion (3.22) is summarized in Theorem 6. 
Theorem 6. The system (3.22) is a "two-time" Hamiltonian system with respect to the Lie-Poisson bracket,

$$
\{A(\gamma) \stackrel{\otimes}{,} A(\mu)\}=[r(\gamma-\mu), A(\gamma) \otimes \mathbf{1}+\mathbf{1} \otimes A(\mu)]
$$

where $A(\gamma) \equiv \Psi_{\gamma} \Psi^{-1}$ and the classical rational $R$-matrix $r(\gamma)$ is equal to $\Pi / \gamma$ with $\Pi$ the permutation operator in $\mathbb{C}^{2} \times \mathbb{C}^{2}$,

$$
\Pi=\left(\begin{array}{llll}
1 & 0 & 0 & 0 \\
0 & 0 & 1 & 0 \\
0 & 1 & 0 & 0 \\
0 & 0 & 0 & 1
\end{array}\right)
$$

The dynamics of the physical fields in the $\xi$ and $\bar{\xi}$-directions are governed by the "matter Hamiltonians",

$$
\begin{aligned}
H^{(\xi)} & :=\frac{1}{\xi-\bar{\xi}} \operatorname{tr} A^{2}(1)=\frac{1}{\xi-\bar{\xi}} \sum_{j, k=1}^{N} \frac{\operatorname{tr}\left(A_{j} A_{k}\right)}{\left(1-\gamma_{j}\right)\left(1-\gamma_{k}\right)}, \\
H^{(\bar{\xi})} & :=\frac{1}{\bar{\xi}-\xi} \operatorname{tr} A^{2}(-1)=\frac{1}{\bar{\xi}-\xi} \sum_{j, k=1}^{N} \frac{\operatorname{tr}\left(A_{j} A_{k}\right)}{\left(1+\gamma_{j}\right)\left(1+\gamma_{k}\right)},
\end{aligned}
$$

and the respective flows generated by $H^{(\xi)}$ and $H^{(\bar{\xi})}$ commute, i.e., $\left\{H^{(\xi)}, H^{(\bar{\xi})}\right\}=0$.

Proof. The main statement can be verified by direct calculation. Commutativity of the Hamiltonians follows from the more general relation

$$
\left\{\operatorname{tr} A^{2}(\gamma), \operatorname{tr} A^{2}(\mu)\right\}=0,
$$

which is valid for arbitrary $\gamma$ and $\mu$. The commutativity of the flows generated by $H^{(\xi)}$ and $H^{(\bar{\xi})}$ is equivalent to the decoupling of the classical equations of motion in (3.22), and may be viewed as a direct consequence of the compatibility of the system (3.22) (cf. Theorem 2). Observe that we have $\left(H^{(\xi)}\right)^{\dagger}=H^{(\bar{\xi})}$,

For the benefit of readers not familiar with the above tensor product notation (see $[13,14]$ for details), we spell out these brackets once more with matrix indices $\alpha, \beta, \ldots=1,2$ indicated explicitly. Setting

$$
(A(\gamma) \otimes A(\mu))_{\alpha \beta, \gamma \delta}:=A(\gamma)_{\alpha \gamma} A(\mu)_{\beta \delta}
$$

and

$$
\Pi_{\alpha \beta, \gamma \delta}=\delta_{\alpha \delta} \delta_{\gamma \beta}
$$

the Poisson brackets (3.53) are equivalent to 


$$
\left\{A_{\alpha \beta}(\gamma), A_{\gamma \delta}(\mu)\right\}=\frac{1}{\gamma-\mu}\left(\delta_{\alpha \delta}(A(\gamma)-A(\mu))_{\gamma \beta}-\delta_{\gamma \beta}(A(\gamma)-A(\mu))_{\alpha \delta}\right) .
$$

Defining

$$
A_{\alpha \beta} \equiv A_{j}^{a} t_{\alpha \beta}^{a},
$$

where $t^{a}$ are the generators (so far of $S L(2, \mathbb{C})$ ) and inserting $(3.11)$ into $(3.53)$, we get

$$
\left\{A_{j} \stackrel{\otimes}{,} A_{k}\right\}=\delta_{j k}\left[I, A_{j} \otimes \mathbf{1}\right]
$$

or, equivalently,

$$
\left\{A_{j}^{a}, A_{k}^{b}\right\}=\delta_{j k} f^{a b} A_{j}^{c},
$$

where $f^{a b}{ }_{c}$ are the structure constants of $S L(2, \mathbb{C})$.

With (3.54) we can thus reexpress the equations for the conformal factor (2.5) in the form

$$
\mathcal{C}^{(\xi)}:=-2 k_{\xi}+H^{(\xi)} \approx 0, \quad \mathcal{C}^{(\bar{\xi})}:=-2 k_{\bar{\xi}}+H^{(\bar{\xi})} \approx 0 .
$$

Thus, in accordance with the general theory of constraints [38], we shall from now on regard (3.58) as constraints à la Dirac rather than merely as equations determining the conformal factor (accordingly, $\approx$ means "weakly zero"). This interpretation is appropriate for generally covariant theories where the local (gauge) invariances give rise to canonical constraints whose "matter parts" are just the conventional Hamiltonians (3.54). More precisely, the constraints (3.58) express the invariance of the theory under local translations in $\xi$ and $\bar{\xi}$, respectively; as such they are linear combinations of the WDW and diffeomorphism constraints corresponding to the invariance of the theory with respect to local translations in "time" $\rho$ and "space" $x$. As before we have a reality condition $\left(\mathcal{C}^{(\xi)}\right)^{\dagger}=\mathcal{C}^{(\bar{\xi})}$ for the constraints.

Interpreting (3.58) as canonical constraints requires that we enlarge the phase space so as to account for the gravitational degrees of freedom (the conformal factor and the dilaton). Their canonical brackets are given by

$$
\left\{\xi, 2 k_{\xi}\right\}=\left\{\bar{\xi}, 2 k_{\bar{\xi}}\right\}=1, \quad\left\{\bar{\xi}, 2 k_{\xi}\right\}=\left\{\xi, 2 k_{\bar{\xi}}\right\}=0 .
$$

(Strictly speaking, the derivation of these brackets would require that we undo the choice of Weyl coordinates, on which (2.1) and (2.4) are based, but we will skip this step here.) Use of (3.59) and some further computation then show that the constraints commute like the matter Hamiltonians in terms of which they are defined,

$$
\left\{\mathcal{C}^{(\xi)}, \mathcal{C}^{(\bar{\xi})}\right\}=0
$$

A noteworthy feature of the new formulation is that the dimension of the system has been effectively reduced from two to one by trading the "space" variable $x$ and the "time" variable $\rho$ for two "time" variables $\xi$ and $\xi$. We can thus regard the spectral parameter currents $A(\gamma)$ at a fixed but arbitrarily chosen base point $\left(x_{0}, \rho_{0}\right)$ as the 
fundamental canonical variables. In other words, instead of considering phase space variables depending on the space coordinates, we now take them to depend on the spectral parameter. Since this point can be chosen at will, this formulation manifestly preserves $2 \mathrm{~d}$ covariance. The "time evolutions" of any phase space function $F\left(\left\{A_{j}\right\} ; \xi, \bar{\xi}, k_{\bar{\xi}}, k_{\xi}\right)$ are then generated as usual by commutation with the constraints $\mathcal{C}^{(\xi)}$ and $\mathcal{C}^{(\bar{\xi})}$, i.e.,

$$
\frac{d F}{d \xi}=\left\{\mathcal{C}^{(\xi)}, F\right\}, \quad \frac{d F}{d \bar{\xi}}=\left\{\mathcal{C}^{(\bar{\xi})}, F\right\} .
$$

It is important that the derivatives appearing here are total derivatives with respect to $\xi$ and $\bar{\xi}$, with the first term of $\mathcal{C}^{(\xi)}$ or $\mathcal{C}^{(\bar{\xi})}$ generating the partial derivatives with respect to the coordinates, and the second term taking care of the $(\xi, \vec{\xi})$-dependence of $A_{j}$. Altogether the action of the constraints on any phase space function is thus simply given by the operators

$$
\mathcal{C}^{(\xi)} \cong \frac{d}{d \xi}, \quad \mathcal{C}^{(\bar{\xi})} \cong \frac{d}{d \bar{\xi}} .
$$

As we will see, this remains true for the quantized theory where the constraints become operators acting on a Hilbert space of wave functionals.

Observables in the sense of Dirac are by definition all those functionals $\mathcal{O}$ on phase space which weakly commute with the constraints $\mathcal{C}^{(\bar{\xi})}$ and $\mathcal{C}^{(\bar{\xi})}$ but do not vanish on the constraint hypersurface $\mathcal{C}^{(\xi)}=\mathcal{C}^{(\bar{\xi})}=0$, i.e.,

$$
\left\{\mathcal{C}^{(\xi)}, \mathcal{O}\right\} \approx 0, \quad\left\{\mathcal{C}^{(\bar{\xi})}, \mathcal{O}\right\} \approx 0 .
$$

By (3.62) the observables are therefore highly nonlocal objects as one would expect on general grounds $[4,5]$. It is easy to see that our model admits an infinite number of independent observables. First of all, the parameters $w_{1}, \ldots, w_{N}$ trivially belong to this class since they commute with everything. Secondly, and more importantly, the monodromies $M_{1}, \ldots, M_{N}$ defined in (3.17) are also observables for arbitrary $N$. This fact is obvious from (3.18), but can also be rephrased in canonical language. Namely, as functionals on phase space, the monodromy matrices depend both on $\left\{A_{j}\right\}$ and $\left\{\gamma_{j}\right\}$; furthermore, $M_{j}$ does not commute with the matter Hamiltonians $H^{(\xi)}$ and $H^{(\bar{\xi})}$ but only with the full constraints $\mathcal{C}^{(\xi)}$ and $\mathcal{C}^{(\bar{\xi})}$ of (3.58); for instance,

$$
\frac{d M_{j}}{d \xi}=\left\{\mathcal{C}^{(\xi)}, M_{j}\right\}=-\left\{2 k_{\xi}, \xi\right\} \frac{\partial M_{j}}{\partial \xi}+\left\{H^{(\xi)}, M_{j}\right\}=\frac{\partial M_{j}}{\partial \xi}+\left\{H^{(\xi)}, M_{j}\right\}=0 .
$$

We here recognize an important difference between dimensionally reduced gravity and the corresponding flat space $\sigma$-models, where $\gamma$ would be coordinate independent and the traces $\operatorname{tr} A(\gamma)^{2}$ would already be constants of motion by (3.55).

All observables can be generated from the set

$$
\text { Obs }:=\left\{w_{1}, \ldots, w_{N} ; M_{1}, \ldots, M_{N}\right\}
$$

by taking arbitrary products and linear combinations. In this sense Obs constitutes a complete set of classical (and quantum) observables for arbitrary $N$. These are the 
conserved "nonlocal charges" of dimensionally reduced gravity. The monodromies are not easy to handle at the canonical level because of their nonlocal dependence on $A(\gamma)$ [39]. For this reason we shall mostly deal with the restricted set of observables

$$
\widetilde{\mathrm{Obs}}:=\left\{w_{1}, \ldots, w_{N} ; \operatorname{tr} A_{1}^{2}, \ldots, \operatorname{tr} A_{N}^{2} ; A_{\infty}\right\} \subset \mathrm{Obs} .
$$

We include $A_{\infty}$ (cf. Lemma 2) here to keep the discussion as general as possible. If $\Psi$ is regular at infinity, the condition $A_{\infty}=0$ should be treated as a first class constraint since this matrix obviously closes into an $S L(2, \mathbb{R})$ algebra and its entries are integrals of motion. The meaning of $A_{\infty}$ is further clarified by the following theorem.

Theorem 7. The matrix elements of $A_{\infty}$ are the canonical generators of the group of Ehlers transformations with respect to the Poisson structure (3.53).

Proof. By (3.51), an infinitesimal Ehlers transformation with parameter $\epsilon_{\alpha \beta}$ acts on $A_{j}$ as

$$
\delta A_{j}=\left[A_{j}, \epsilon\right] .
$$

On the other hand, we have

$$
\left\{\epsilon_{\gamma \delta}\left(A_{\infty}\right)_{\delta \gamma},\left(A_{j}\right)_{\alpha \beta}\right\}=\left[A_{j}, \epsilon\right]_{\alpha \beta}
$$

by (3.57).

At this point the following comments concerning the status of the constraints (3.40) and (3.50) are in order. In the sequel we shall mainly deal with the case $\gamma_{j}=-\bar{\gamma}_{j}$ (i.e., set $m=n$ in (3.47)). Then condition (3.40) just means that $A_{j} \in \operatorname{sl}(2, \mathbb{R})$ and the Poisson bracket (3.57) is the standard Kirillov-Konstant bracket for $\operatorname{sl}(2, \mathbb{R})$. The asymptotic regularity condition $A_{\infty}=0$ should be considered as a part of the coset constraints. It ensures the symmetry of $\Psi$ with respect to the involution $\gamma \rightarrow \gamma^{-1}$; regularity of $\Psi$ at $\gamma=\infty$ then follows from the assumed regularity of $\Psi$ at $\gamma=0$. However, the constraint (3.50) is not an ordinary phase space constraint since it relates the phase space variables at the different "times" $\gamma_{j}$.

Let us mention two alternative ways of writing the brackets (3.53).

- The total phase space of the theory may also be parametrized by the coefficients of the expansion of $A(\gamma)$ at $\gamma=\infty$,

$$
A(\gamma)=\sum_{k=1}^{\infty} \gamma^{-k} \widetilde{A}_{k}, \quad \widetilde{A}_{k} \equiv \sum_{j=1}^{N} A_{j} \gamma_{j}^{k-1} .
$$

Assuming the total phase space to be

$$
\mathcal{H}=\left\{\widetilde{A_{k}} \mid k=1,2, \ldots\right\}
$$

and substituting (3.66) into (3.53), we get

$$
\left\{\widetilde{A}_{j}^{a}, \widetilde{A}_{k}^{b}\right\}=f^{a b}{ }_{c} \widetilde{A}_{j+k}^{c}, \quad j, k>1,
$$


i.e., "half" of the current algebra $A_{1}^{(1)} \equiv s \widehat{s(2, \mathbb{C})}$.

For the isomonodromic sectors with finite $N$, the currents $\widetilde{A}_{j}$ are clearly dependent. For $N \rightarrow \infty$, on the other hand, they can be considered as independent canonical variables; for arbitrary non-isomonodromic solutions the natural way to generalize our present construction might thus be in terms of the variables $\widetilde{A}_{j}$ together with (3.67). The Ehlers charge coincides with the first term of the expansion (3.66), i.e., $-A_{\infty} \equiv \widetilde{A}_{1}$.

- For the infinite set of currents,

$$
\begin{aligned}
J_{m, n} & \equiv \sum_{j=1}^{N} \frac{A_{j}}{\left(1-\gamma_{j}\right)^{m}\left(1+\gamma_{j}\right)^{n}} \\
J_{1,0} & =\frac{\xi-\bar{\xi}}{2} g_{\xi} g^{-1}, \quad J_{0,1}=\frac{\bar{\xi}-\xi}{2} g_{\bar{\xi}} g^{-1},
\end{aligned}
$$

we have the "current algebra"

$$
\left\{J_{m, n}^{a}, J_{m^{\prime}, n^{\prime}}^{b}\right\}=f^{a b}{ }_{c} J_{m+m^{\prime}, n+n^{\prime}}^{c} .
$$

Now $-A_{\infty}$ coincides with $J_{0,0}$.

The Poisson structure (3.53) also appears in Chern-Simons theory, where one starts from the connection

$$
\mathcal{A}:=A_{1}(\gamma, \bar{\gamma}) d \gamma+A_{2}(\gamma, \bar{\gamma}) d \bar{\gamma},
$$

with the bracket

$$
\left\{A_{1}^{a}(\gamma, \bar{\gamma}), A_{2}^{b}(\mu, \bar{\mu})\right\}=\delta^{a b} \delta^{2}(\gamma-\mu) .
$$

Imposing the flatness condition

$$
A_{1 \grave{\gamma}}-A_{2 \gamma}+\left[A_{1}, A_{2}\right]=0
$$

and choosing a holomorphic gauge $A_{2}=0$ (see [40]), one can derive the bracket (3.53) as the Dirac bracket for the remaining component $A(\gamma) \equiv A_{1}(\gamma)$, at least for the punctured sphere. This link is discussed in more detail in [41,39].

To conclude this section we would like to briefly comment on the relation between the new Hamiltonian formulation and the conventional one based on the use of one space and one time variable, where $g(\xi, \bar{\xi})$ would be treated as a quantum field coupled to $2 \mathrm{~d}$ gravity and a dilaton. It will be sufficient to explain the differences for the principal chiral model (see, e.g., [42] for a description of the corresponding flat space model). There the main objects of interest are the $x$-dependent currents at fixed "time" $\rho$,

$$
J_{\rho}=\rho g_{\rho} g^{-1} \quad \text { and } \quad J_{x}=\rho g_{x} g^{-1}
$$

(which are obviously related to the currents $J_{0,1}$ and $J_{1,0}$ from (3.68)) and their equal time Poisson brackets, which read 


$$
\begin{aligned}
& \left\{J_{\rho}(x) \stackrel{\otimes}{,} J_{\rho}(y)\right\}=\rho\left[\Pi, J_{\rho}(x) \otimes \mathbf{1}\right] \delta(x-y), \\
& \left\{J_{\rho}(x) \stackrel{\otimes}{,} J_{x}(y)\right\}=\rho\left[\Pi, J_{x}(x) \otimes \mathbf{1}\right] \delta(x-y)+\rho \Pi \delta^{\prime}(x-y), \\
& \left\{J_{x}(x) \stackrel{\otimes}{,} J_{x}(y)\right\}=0
\end{aligned}
$$

(for coset space $\sigma$-models the matrix $\Pi$ would not be independent of the fields). The Hamiltonian determining the evolution in the $\rho$-direction is

$$
H=\int \rho^{-1} \operatorname{tr}\left(-J_{\rho}^{2}+J_{x}^{2}\right) d x .
$$

The non-ultralocal $\delta^{\prime}$-term in (3.71) has been a notorious source of trouble, leading to irresoluble ambiguities in the Poisson brackets of certain integrated phase space quantities [42-44]. It also represents a serious obstacle towards the application of standard quantization techniques $[13,14]$. By contrast, our "two-time" formalism sidesteps this difficulty, as the troublesome non-ultralocal term has disappeared. Besides yielding the same canonical equations of motion as the usual approach, the (real) Hamiltonian defining the evolution in $\rho$-direction in our formalism is equal to (recall from (2.11) that $\rho=\operatorname{Im} \xi$ )

$$
\begin{aligned}
H^{(\rho)} & =-\frac{1}{2} i\left(H^{(\xi)}-H^{(\bar{\xi})}\right)=\frac{1}{4} \rho\left(\operatorname{tr}\left(g_{\xi} g^{-1}\right)^{2}+\operatorname{tr}\left(g_{\bar{\xi}} g^{-1}\right)^{2}\right) \\
& =\frac{1}{2} \rho \operatorname{tr}\left(-\left(g_{\rho} g^{-1}\right)^{2}+\left(g_{x} g^{-1}\right)^{2}\right)
\end{aligned}
$$

and therefore agrees with the Hamiltonian density (3.72) of the usual approach. Let us note that the minus sign in front of $J_{\rho}^{2}$ is simply due to the "Wick rotation" of the $1+1$ metric to a $0+2$ metric; the apparent lower unboundedness of the Hamiltonian (3.73) is therefore spurious.

\subsection{Relation to the Schlesinger equations}

Our equations (3.22) are closely related to the so-called Schlesinger equations [45] which play an important role in the theory of integrable systems [26]. To exhibit the relation, let us consider $\gamma_{j}, j=1, \ldots, N$ as independent deformation parameters and suppose that the monodromy data $\left\{T_{j}, C_{j}\right\}$ are $\gamma_{j}$-independent. Instead of (3.12) and (3.1), we would then get the following deformation equations in $\gamma_{j}$ :

$$
\frac{\partial \Psi}{\partial \gamma_{j}}=-\frac{A_{j}}{\gamma-\gamma_{j}} \Psi, \quad j=1, \ldots, N
$$

Demanding compatibility of (3.74) and (3.12) we arrive at the classical Schlesinger equations [26],

$$
\frac{\partial A_{j}}{\partial \gamma_{k}}=\frac{\left[A_{j}, A_{k}\right]}{\gamma_{j}-\gamma_{k}} \quad(k \neq j) ; \quad \frac{\partial A_{j}}{\partial \gamma_{j}}=-\sum_{i \neq j} \frac{\left[A_{j}, A_{i}\right]}{\gamma_{j}-\gamma_{i}}
$$


The system (3.75) is an $N$-time Hamiltonian system with respect to the Poisson structure (3.53) [26] with times $\gamma_{j}$ for the Hamiltonians,

$$
H_{j}=\sum_{i \neq j} \frac{\operatorname{tr}\left(A_{i} A_{j}\right)}{\gamma_{j}-\gamma_{i}}, \quad j=1, \ldots, N
$$

The Hamiltonians $H_{j}$ mutually commute and can alternatively be obtained from

$$
H_{j}=\left.\operatorname{res}\right|_{\gamma=\gamma_{j}} \operatorname{tr} A^{2}(\gamma) .
$$

The $\tau$-function (3.28) is the generating function for Hamiltonians $H_{j}$ in the sense that

$$
\frac{\partial \tau}{\partial \gamma_{j}}=H_{j} \tau
$$

Now we are in the position to formulate the theorem relating the Schlesinger equations (3.75) to our deformation equations (3.22).

Theorem 8. Let the functions $A_{j}\left(\left\{\gamma_{k}\right\}\right), j=1, \ldots, N$ solve the Schlesinger equations (3.75) and obey the constraint (3.15). Furthermore, let the variables $\gamma_{j}$ depend on $(\xi, \bar{\xi})$ according to (3.3), i.e., $\gamma_{j}=\gamma\left(w_{j} ; \xi, \bar{\xi}\right)$. Then the residue functions $A_{j}\left(\left\{\gamma_{k}(\xi, \bar{\xi})\right\}\right)$ satisfy Eqs. (3.22).

Proof. The proof is straightforward. Using (3.75), (3.2), we get, for example,

$$
\begin{aligned}
A_{j \xi} & =\sum_{k} \frac{\partial A_{j}}{\partial \gamma_{k}} \gamma_{k \xi}=\frac{1}{\xi-\bar{\xi}} \sum_{k \neq j} \frac{\left[A_{j}, A_{k}\right]}{\gamma_{j}-\gamma_{k}}\left\{\gamma_{k} \frac{1+\gamma_{k}}{1-\gamma_{k}}-\gamma_{j} \frac{1+\gamma_{j}}{1-\gamma_{j}}\right\} \\
& =\frac{2}{\xi-\bar{\xi}} \sum_{k \neq j} \frac{\left[A_{k}, A_{j}\right]}{\left(1-\gamma_{j}\right)\left(1-\gamma_{k}\right)}+\frac{1}{\xi-\bar{\xi}}\left[A_{j}, \sum_{k} A_{k}\right] .
\end{aligned}
$$

The constraint (3.15) eliminates the last term in (3.79).

To clarify the link between Hamiltonians $H^{(\xi)}, H^{(\bar{\xi})}$ (3.54) and the Hamiltonians $H_{j}$ (3.76), we note that the evolution in the $\xi$-direction of an arbitrary solution of (3.75) is given by the Hamiltonian

$$
\sum_{j} H_{j} \gamma_{j \xi}=\frac{1}{\xi-\bar{\xi}} \sum_{k \neq j} \frac{\operatorname{tr}\left(A_{j} A_{k}\right)}{\left(1-\gamma_{j}\right)\left(1-\gamma_{k}\right)}-\frac{1}{2(\xi-\bar{\xi})} \sum_{k \neq j} \operatorname{tr}\left(A_{j} A_{k}\right) .
$$

Using (3.26) and comparing this result with (3.54), we get

$$
H^{(\xi)}=\sum_{j} H_{j} \gamma_{j \xi}-\frac{1}{\xi-\bar{\xi}} \sum_{j} \operatorname{tr} A_{j}^{2}\left\{\frac{1}{2}-\frac{1}{\left(1-\gamma_{j}\right)^{2}}\right\}+\frac{1}{2(\xi-\bar{\xi})} \operatorname{tr}\left(A_{\infty}\right)^{2}
$$

Since the terms containing tr $A_{j}^{2}$ commute with all $A_{k}$ by virtue of (3.53), they do not give any contribution to the equations of motion, and thus can be interpreted as 
contributing to the "vacuum energy" only (the last term on the r.h.s. obviously vanishes for asymptotically flat solutions). Upon quantization the "vacuum energy terms" turn into Casimir operators, and do contribute to the wave function via an explicitly computable phase factor.

The Schlesinger equations together with (3.3) imply that the dependence of $A_{j}$ (and in fact any phase space variable depending on the $A_{j}$ 's) on the parameters $w_{j}$ is governed by the mutually commuting Hamiltonians,

$$
H^{\left(w_{j}\right)}=\frac{\partial \gamma_{j}}{\partial w_{j}} H_{j},
$$

since

$$
\frac{\partial A_{j}}{\partial w_{k}}=\left\{H^{\left(w_{k}\right)}, A_{j}\right\} .
$$

Therefore the Hamiltonian $H^{\left(w_{j}\right)}$ (3.81) can be interpreted as a generator of translations in the variable $w_{j}$, as it moves the position of the $j$ th singularity. The Hamiltonians (3.81) also commute with the total Hamiltonians $H^{(\xi)}$ and $H^{(\bar{\xi})}$ provided (3.15) is satisfied. In fact, (3.82) means that, in analogy to evolution in $\xi$ and $\bar{\xi}$ directions, the proper treatment of the evolution in $w_{j}$ direction is the following: the canonically conjugate variable to $w_{j}$ is $2 k_{w_{j}}$; then the equation $2 k_{w_{j}}-H^{\left(w_{j}\right)}=0$ should be treated as a constraint in analogy with the constraints $\mathcal{C}^{(\bar{\xi})}$ and $\mathcal{C}^{(\bar{\xi})}$ (3.58).

\section{Quantization}

\subsection{Commutation relations and Bethe ansatz}

To quantize the model, we replace the Poisson brackets (3.53) by commutators in the usual fashion,

$$
[A(\gamma) \stackrel{\otimes}{,} A(\mu)]=i \hbar[r(\gamma-\mu), A(\gamma) \otimes \mathbf{1}+\mathbf{1} \otimes A(\mu)] .
$$

The entries of the matrix of $A(\gamma)$ thus become operators acting on a Hilbert space to be specified below; note that on the l.h.s. of (4.1) we have a commutator of operators in Hilbert space whereas on the r.h.s. we have a commutator of ordinary matrices. This means in particular that the expansion (3.12) is no longer valid as an operator statement, but must be reinterpreted as a property of the states on which $A(\gamma)$ acts. We write

$$
A(\gamma) \equiv \frac{i \hbar}{2}\left(\begin{array}{cc}
\mathbf{h}(\gamma) & 2 \mathbf{e}(\gamma) \\
2 \mathbf{f}(\gamma) & -\mathbf{h}(\gamma)
\end{array}\right)
$$

The reality constraint (3.40) translates into

$$
\mathbf{h}(\gamma)^{\dagger}=\mathbf{h}(-\bar{\gamma}), \quad \mathbf{e}(\gamma)^{\dagger}=\mathbf{e}(-\bar{\gamma}), \quad \mathbf{f}(\gamma)^{\dagger}=\mathbf{f}(-\bar{\gamma}) .
$$

(4.1) and (4.2) yield the following commutation relations for the operators $\mathbf{h}(\gamma), \mathbf{e}(\gamma)$ and $\mathbf{f}(\gamma)$ : 


$$
\begin{aligned}
{\left[\mathbf{h}(\gamma), \mathbf{e}\left(\gamma^{\prime}\right)\right] } & =-\frac{2}{\gamma-\gamma^{\prime}}\left(\mathbf{e}(\gamma)-\mathbf{e}\left(\gamma^{\prime}\right)\right), \\
{\left[\mathbf{h}(\gamma), \mathbf{f}\left(\gamma^{\prime}\right)\right] } & =\frac{2}{\gamma-\gamma^{\prime}}\left(\mathbf{f}(\gamma)-\mathbf{f}\left(\gamma^{\prime}\right)\right), \\
{\left[\mathbf{e}(\gamma), \mathbf{f}\left(\gamma^{\prime}\right)\right] } & =-\frac{1}{\left(\gamma-\gamma^{\prime}\right)}\left(\mathbf{h}(\gamma)-\mathbf{h}\left(\gamma^{\prime}\right)\right),
\end{aligned}
$$

with all other commutators equal to zero. For coincident arguments we have, for instance,

$$
[\mathbf{h}(\gamma), \mathbf{e}(\gamma)]=-2 \frac{d \mathbf{e}(\gamma)}{d \gamma}
$$

The Hamiltonians (3.54) remain unchanged; they can be written out more explicitly in terms of the matrix elements of $A(\gamma)$ and thereby cast into a form reminiscent of the Sugawara construction. Explicitly,

$$
\begin{aligned}
H^{(\xi)} & =\frac{1}{\xi-\bar{\xi}} \operatorname{tr}\left(A^{2}(1)\right) \equiv-\frac{\hbar^{2}}{\xi-\bar{\xi}}\left(\frac{1}{2} \mathbf{h}(1) \mathbf{h}(1)+\mathbf{e}(1) \mathbf{f}(1)+\mathbf{f}(1) \mathbf{e}(1)\right), \\
H^{(\bar{\xi})} & =-\frac{1}{\xi-\xi} \operatorname{tr}\left(A^{2}(-1)\right) \\
& \equiv \frac{\hbar^{2}}{\xi-\bar{\xi}}\left(\frac{1}{2} \mathbf{h}(-1) \mathbf{h}(-1)+\mathbf{e}(-1) \mathbf{f}(-1)+\mathbf{f}(-1) \mathbf{e}(-1)\right) .
\end{aligned}
$$

As already mentioned, we shall restrict ourselves to states on which the operator $A(\gamma)$ may be represented as in (3.12) with $\gamma_{j}=-\bar{\gamma}_{j}$. Accordingly, we put

$$
A_{j} \equiv \frac{i \hbar}{2}\left(\begin{array}{cc}
\mathbf{h}_{j} & 2 \mathbf{e}_{j} \\
2 \mathbf{f}_{j} & -\mathbf{h}_{j}
\end{array}\right),
$$

so that

$$
\mathbf{h}(\gamma)=\sum_{j=1}^{N} \frac{\mathbf{h}_{j}}{\gamma-\gamma_{j}}, \quad \mathbf{e}(\gamma)=\sum_{j=1}^{N} \frac{\mathbf{e}_{j}}{\gamma-\gamma_{j}}, \quad \mathbf{f}(\gamma)=\sum_{j=1}^{N} \frac{\mathbf{f}_{j}}{\gamma-\gamma_{j}} .
$$

The operators $\mathbf{h}_{j}, \mathbf{e}_{j}$ and $\mathbf{f}_{j}$ are the anti-Hermitian Chevalley generators of $S L(2, \mathbb{R}$ ) (see appendix) obeying the standard commutation relations

$$
\left[\mathbf{h}_{j}, \mathbf{e}_{j}\right]=2 \mathbf{e}_{j}, \quad\left[\mathbf{h}_{j}, \mathbf{f}_{j}\right]=-2 \mathbf{f}_{j}, \quad\left[\mathbf{e}_{j}, \mathbf{f}_{j}\right]=\mathbf{h}_{j},
$$

as a consequence of (4.4).

For the explicit construction of solutions it is convenient to switch from the $S L(2, \mathbb{R})$ basis to an $S U(1,1)$ basis in terms of which raising and lowering operators can be defined; this also facilitates the comparison between the noncompact case $G=S U(1,1)$ and the compact case $G=S U(2)$. The $S U(1,1)$ Chevalley generators are defined by

$$
e_{j}:=\frac{1}{2}\left(-i \mathbf{h}_{j}+\mathbf{e}_{j}+\mathbf{f}_{j}\right), \quad f_{j}:=\frac{1}{2}\left(i \mathbf{h}_{j}+\mathbf{e}_{j}+\mathbf{f}_{j}\right), \quad h_{j}:=i\left(\mathbf{f}_{j}-\mathbf{e}_{j}\right) .
$$

They also obey

$$
\left[h_{j}, e_{j}\right]=2 e_{j}, \quad\left[h_{j}, f_{j}\right]=-2 f_{j}, \quad\left[e_{j}, f_{j}\right]=h_{j},
$$


but are no longer anti-Hermitean, satisfying instead the following hermiticity properties:

$$
h_{j}^{\dagger}=h_{j}, \quad e_{j}^{\dagger}=-f_{j} .
$$

For $S U(2)$, the operators are the same but the hermiticity condition reads

$$
h_{j}^{\dagger}=h_{j}, \quad e_{j}^{\dagger}=+f_{j} .
$$

In both cases we can interpret $e_{j}$ and $f_{j}$ as creation and annihilation operators, respectively (or vice versa) and diagonalize the operators $h_{j}$.

Physical states based on unitary representations of $S U(2)$ or on the discrete series representations of $S U(1,1)$ always admit a "ground state" $|p\rangle$ labeled by some analytic function $p=p(\gamma)$ subject to the reality condition $\overline{p(\gamma)}=p(-\bar{\gamma})$. This state is a lowest weight state in the sense that it is assumed to obey the conditions

$$
h(\gamma)|p\rangle=p(\gamma)|p\rangle \quad \text { and } \quad f(\gamma)|p\rangle=0 .
$$

The classical expansion (3.12) corresponds to the special choice

$$
p(\gamma)=\sum_{j=1}^{N} \frac{s_{j}}{\gamma-\gamma_{j}},
$$

which is equivalent to $h_{j}|p\rangle=s_{j}|p\rangle$. The "excited states" are obtained by applying the raising operators to $|p\rangle$. More specifically, we define the (off-shell) Bethe states for both $S U(2)$ and $S U(1,1)$ by

$$
\left|p ; v_{1}, \ldots, v_{M}\right\rangle:=e\left(v_{1}\right) \ldots e\left(v_{M}\right)|p\rangle,
$$

where $e(\gamma)$ is the analog of (4.8) and the complex parameters $v_{i}$ are arbitrary at this point. Thus the operators $e(\gamma)$ and $f(\gamma)$ indeed play the role of creation and annihilation operators, respectively, as asserted above; interchanging them turns the representation "upside down". For $S U(2)$, the possible values for $s_{j}$ are negative integer (since we are working with lowest weight states) and the spectrum of $h_{j}$ (and hence the number of excitations in (4.16)) is bounded with eigenvalues $-\left|s_{j}\right|,-\left|s_{j}\right|+1, \ldots,\left|s_{j}\right|$. For the discrete representations of $S U(1,1), s_{j}$ is either positive integer with $s_{j} \geq 2$ and a semi-infinite "topless" spectrum $s_{j}, s_{j}+1, \ldots$, or negative integer with $s_{j} \leq 0$ and a semi-infinite "bottomless" spectrum with $s_{j}, s_{j}-1, \ldots$ The eigenvalue of the Casimir operator is always $s_{j}\left(s_{j}-2\right)$ in these conventions. For these representations, the physical parameters (masses, etc.) are quantized, and we can claim that the quantum theory is "more regular" than the classical theory because the quantization of $s_{j}$ severely constrains the types of singularity that can appear in the quantum wave functional. For the principal and supplementary series of $S U(1,1)$, on the other hand, which have no $S U(2)$ analog, $s_{j}$ is a continuous parameter, and there is consequently no quantization of physical parameters. (4.15) and the first relation in (4.14) still hold but the Bethe ansatz (4.16) no longer works because the corresponding ground state does not exist.

For the full theory we must also quantize the gravitational degrees of freedom $2 k_{\xi}$, $2 k_{\bar{\xi}}$ and $\xi, \bar{\xi}$, keeping in mind that these are really fields in a special gauge and not just 
coordinates on the worldsheet. We must therefore replace the Poisson brackets (3.59) by commutators and construct an operator representation for the gravitational phase space variables. From the canonical brackets (3.59) we deduce

$$
\left[\xi, 2 k_{\xi}\right]=\left[\bar{\xi}, 2 k_{\bar{\xi}}\right]=i \hbar, \quad\left[\bar{\xi}, 2 k_{\xi}\right]=\left[\xi, 2 k_{\bar{\xi}}\right]=\left[2 k_{\xi}, 2 k_{\bar{\xi}}\right]=0 .
$$

To realize these commutation relations, we take

$$
2 k_{\xi}=-i \hbar \frac{\partial}{\partial \xi}+f(\xi, \bar{\xi}), \quad 2 k_{\bar{\xi}}=-i \hbar \frac{\partial}{\partial \bar{\xi}}+\bar{f}(\xi, \bar{\xi}),
$$

with an arbitrary function $f$ satisfying

$$
\frac{\partial f}{\partial \bar{\xi}}=\frac{\partial \bar{f}}{\partial \xi}
$$

A different choice of $f$ will result in a renormalization of the physical states by a function of the coordinates and will not affect the physical content of the theory; without loss of generality we can thus set

$$
f(\xi, \bar{\xi})=0 \text {. }
$$

The main advantage of (4.17) is that by representing $(\xi, \bar{\xi})$ as multiplication operators we salvage their interpretation as coordinates; otherwise the spectral parameter $\gamma$ would not remain a function but become a nonlocal differential operator and thus very awkward to deal with. It is then obvious that the two equations (4.24) are mutually compatible for the same reason that their classical counterparts (2.7) are. Recall that the worldsheet coordinates $(\xi, \bar{\xi})$ appear explicitly only because we have adopted the special gauge (2.11) identifying the dilaton field with one of the coordinates. In other words, this choice of gauge makes the quantum state $\Phi$ time-dependent through the identification of time with the "clock field" $\rho$. We note that this long suspected mechanism for the emergence of time from the "timeless" WDW equation here comes almost for free (see, e.g., [46] for a review and further references). In a covariant treatment the gauge choice (2.11) would have to be undone, and the full quantum state would be a functional of $\rho$ rather than a function of the worldsheet coordinates.

\subsection{Hilbert space, physical states and quantum observables}

For obvious reasons we require the total physical Hilbert space $\mathcal{H}$ to be unitary ${ }^{4}$. Accordingly, in a given isomonodromic sector we assign to every $\gamma_{j}$ some unitary representation space of $S L(2, \mathbb{R})$ and then take the direct product of these spaces. We recall that all $\gamma_{j}$ are assumed to be purely imaginary; admitting arbitrary complex $\gamma_{j}$ would necessitate replacing $S L(2, \mathbb{R})$ by $S L(2, \mathbb{C})$, since for non-self-conjugate pairs with $\gamma_{j}=-\bar{\gamma}_{k}\left(\gamma_{j} \neq \gamma_{k}\right)$ the matrices $A_{j}$ are complex. As is well known,

\footnotetext{
${ }^{4}$ Although in an Euclidean formulation the requirement of unitarity should really be replaced by some version of the Osterwalder-Schrader reflection positivity.
} 
unitary representations of noncompact groups are infinite dimensional. For $\operatorname{SL}(2, \mathbb{R})$ (or $S U(1,1)$ ) one distinguishes the continuous (principal and supplementary) series and the discrete series representations [47] (see appendix for a summary), whereas for $S L(2, \mathbb{C})$ no discrete unitary representations exist. In contradistinction to the compact case, where we would have to deal with the standard spin $s$ representations of $S U(2)$ utilized in [2], we must decide therefore which unitary representations of $\operatorname{SL}(2, \mathbb{R})$ to use here. Although we will be mainly concerned with discrete series representations in the remainder, we would like to emphasize that our only reason for ignoring the continuous series representations here is that the technology for solving $\mathrm{KZ}$ equations for them is not yet sufficiently developed. As we just explained, the necessity of including such representations becomes already evident when one tries to extend the present treatment to arbitrary complex $\gamma_{j}$.

The total Hilbert space containing all $N$-soliton sectors is obviously quite large. As explained above, we can view the quantized $N$-soliton sector as a system of $N$ compact (for $S U(2)$ ) or noncompact (for $S U(1,1)$ ) "spins" located at the points $(x, \rho)=$ $\left(w_{j}, 0\right)$, where the classical solutions of (2.4) and (2.7) generically have singularities on the worldsheet [28]. Since we expect all worldsheet points to be equivalent, it seems that we would have to assume these representations to be the same for all $w_{j}$. However, such configurations would not give all possible classical solutions in the classical limit. This suggests that we should not assign one particular representation to every point, but instead the (formal) direct sum of all representations,

$$
\mathcal{H}\left(w_{j}\right):=\bigoplus_{s} \mathcal{H}^{(s)}
$$

(of $S U(2)$ or $S U(1,1)$ if $w_{j}$ is real and $S L(2, \mathbb{C})$ otherwise). Then the Hilbert space associated with an isomonodromic sector is parametrized by the set $\left(w_{1}, \ldots, w_{N}\right)$ (which is invariant under complex conjugation) and given by the direct product

$$
\mathcal{H}^{(N)}\left(\left\{w_{j}\right\}\right):=\bigotimes_{j=1}^{N} \mathcal{H}\left(w_{j}\right)
$$

The full Hilbert space $\mathcal{H}$ should contain all these spaces as subspaces, i.e., we should demand that

$$
\mathcal{H}^{(N)}\left(\left\{w_{j}\right\}\right) \subset \mathcal{H}
$$

for all possible choices of $N$ and $w_{j}$. For any two disjoint sets of data $\left\{w_{j}\right\}$ and $\left\{w_{k}^{\prime}\right\}$ with respective soliton numbers $N$ and $N^{\prime}$, we can construct a new Hilbert space describing both configurations by taking the direct product

$$
\left.\mathcal{H}^{\left(N+N^{\prime}\right)}\left(w_{j}, w_{k}^{\prime}\right\}\right):=\mathcal{H}^{(N)}\left(\left\{w_{j}\right\}\right) \bigotimes \mathcal{H}^{\left(N^{\prime}\right)}\left(\left\{w_{k}^{\prime}\right\}\right)
$$

If the sets are not disjoint, we simply include the overlapping factors only once in the product. In this way we ensure that the various subspaces are consistently embedded in a partially ordered sequence of Hilbert spaces, and we can therefore define the total 
Hilbert space $\mathcal{H}$ as the inductive limit of the subspaces contained in it. Superficially, $\mathcal{H}$ thus looks like a Fock space with $N$ playing the role of a particle number operator, but matters are complicated by the fact that there is a continuous infinity of parameters on which $\mathcal{H}$ may depend, so we cannot expect the Hilbert space $\mathcal{H}$ to be separable. This construction would be reminiscent of similar constructions in the context of recent work on the loop representation of canonical gravity [48]; at the classical level it is related to the problem of whether and in what sense the isomonodromic solutions exhaust the full phase space of dimensionally reduced Einstein gravity. In summary, the structure of the full Hilbert space $\mathcal{H}$ is rather complicated and remains to be fully elucidated.

Physical states $\Phi$ must satisfy the quantum analog of (3.58), i.e., the WDW equations

$$
\mathcal{C}^{(\xi)} \boldsymbol{\Phi}=\mathcal{C}^{(\bar{\xi})} \Phi=0
$$

By (3.62) these equations are equivalent to

$$
\frac{d \Phi}{d \xi}=\frac{d \Phi}{d \bar{\xi}}=0
$$

Therefore the physical states are independent of the coordinates; we thus have a rather simple realization of the idea that physical states in quantum gravity should be invariant under the full set of $2 \mathrm{~d}$ coordinate transformations! The usefulness of this observation relies essentially on the fact that the coordinate dependence enters essentially only via the spectral parameter $\gamma$ and the constraints $\mathcal{C}^{(\xi)}$ and $\mathcal{C}^{(\bar{\xi})}$ are represented by highly nontrivial operators. Quantum observables $\mathcal{O}$ by definition commute weakly with the constraint operators $\mathcal{C}^{(\xi)}$ and $\mathcal{C}^{(\tilde{\xi})}$, i.e.,

$$
\left[\mathcal{C}^{(\xi)}, \mathcal{O}\right] \Phi=\left[\mathcal{C}^{(\bar{\xi})}, \mathcal{O}\right] \Phi=0
$$

for any physical state $\Phi$. Thus from any such state we can obtain another physical state by application of the operator $\mathcal{O}$. In Section 3.5 we have given a large variety of classical observables based on the monodromy matrices $M_{j}$. Since these present no ordering problems of any kind for finite $N$, we can straightforwardly take them over to the quantum theory. In other words, the quantum monodromies are obtained from (3.19) by promoting $A(\gamma)$ to an operator in accordance with (4.2). As before, we shall consider only the restricted set $\widetilde{\mathrm{Obs}}$ consisting of (the quantum versions of) the classical integrals of motion $\operatorname{tr} A_{j}^{2}$ and $A_{\infty}$. As we will see, the quantization of the Schlesinger equations in terms of $A_{j}$ leads to the $\mathrm{KZ}$ equations; then the quantum monodromies turn out to coincide with the monodromies of the $\mathrm{KZ}$ equations up to similarity transformations and to carry representations of a certain quantum group [49,50].

Since we are working with unitary representations, the natural scalar product on any subspace $\mathcal{H}^{(N)}$ is automatically positive definite (negative norm states would, however, arise in a fully covariant treatment, i.e., prior to the conformal gauge fixing (2.11), and would have to be eliminated by a suitable gauge constraint). Furthermore, owing to the coordinate independence of the physical states (4.21), this scalar product is invariant under the full diffeomorphism group when restricted to physical states because for two such states, 


$$
\frac{d}{d \xi}\left\langle\Phi_{1} \mid \Phi_{2}\right\rangle=\left\langle\mathcal{C}^{(\bar{\xi})} \Phi_{1} \mid \Phi_{2}\right\rangle+\left\langle\Phi_{1} \mid \mathcal{C}^{(\xi)} \Phi_{2}\right\rangle=0
$$

In a covariant formulation we would have to integrate over the fields $\xi(z)$ and $\bar{\xi}(\bar{z})$ with a suitable measure, which would presumably include a Faddeev-Popov determinant to ensure diffeomorphism invariance, together with a $\delta$-functional for gauge fixing. Given observables $\mathcal{O}_{1}, \ldots, \mathcal{O}_{n} \in \widetilde{\mathrm{Obs}}$ and any nontrivial physical state $\Phi$, we can thus compute the correlators

$$
\left\langle\mathcal{O}_{1} \cdots \mathcal{O}_{n}\right\rangle=\frac{\left\langle\Phi\left|\mathcal{O}_{1} \cdots \mathcal{O}_{n}\right| \Phi\right\rangle}{\langle\Phi \mid \Phi\rangle}
$$

By construction, such expectation values are invariant under $2 d$ diffeomorphisms and therefore meaningful objects in quantum gravity.

\subsection{Solving the Wheeler-DeWitt equation}

The central task is now to solve the quantum constraints (4.21), which can be written out as

$$
\left(2 k_{\xi}-H^{(\bar{\xi})}\right) \Phi=\left(2 k_{\bar{\xi}}-H^{(\bar{\xi})}\right) \Phi=0,
$$

where $\Phi$ is the full quantum state. Using (4.17), Eqs. (4.24) take the form

$$
-i \hbar \frac{\partial \Phi}{\partial \xi}=H^{(\xi)} \Phi, \quad-i \hbar \frac{\partial \Phi}{\partial \bar{\xi}}=H^{(\bar{\xi})} \Phi,
$$

where, in the $N$-soliton sector, $\Phi$ is an $\mathcal{H}^{(N)}$-valued function of $(\xi, \bar{\xi})$. Readers may wonder at this point why these equations are first order, since the usual WDW equation is a second order (functional) differential equation. This feature is explained by the fact that the first order equations (4.25) arise due to the separation of the theory into left and right moving sectors, and is similar to the fact that the Virasoro constraints of string theory in the lightcone gauge become linear in the longitudinal target space coordinate $X^{-}$.

To simplify matters we shall not utilize the full Hilbert space (4.19) but only consider functionals that live in the $N$ soliton subspace,

$$
\mathcal{H}^{(N)}\left(\left\{s_{j}, w_{j}\right\}\right):=\bigotimes_{j=1}^{N} \mathcal{H}_{j}, \quad \mathcal{H}_{j} \equiv \mathcal{H}^{\left(s_{j}\right)},
$$

with fixed $w_{j} \in \mathbb{R}$ and $s_{j}$. For the rest of this and the following sections we will work entirely with the $S U(1,1)$ basis from now, because this permits an easy passage to the compact group $S U(2)$. Furthermore, we will restrict attention to the discrete unitary representations, leaving the consideration of continuous representations for future work. Explicit solutions of (4.25) on the subspace $\mathcal{H}^{(N)}$ may be obtained by exploiting the close link between (4.25) and the $\mathrm{KZ}$ equations [15] for $S L(2, \mathbb{R})$, which read 


$$
\frac{\partial \Phi_{\mathrm{KZ}}}{\partial \gamma_{j}}=-i \hbar \sum_{k \neq j} \frac{\Omega_{j k}}{\gamma_{j}-\gamma_{k}} \Phi_{\mathrm{KZ}},
$$

with an $\mathcal{H}^{(N)}$-valued function $\Phi_{\mathrm{KZ}}(\xi, \bar{\xi})$. Here

$$
\Omega_{j k}:=\frac{1}{2} h_{j} \otimes h_{k}+f_{j} \otimes e_{k}+e_{j} \otimes f_{k}
$$

is a linear operator which for $j \neq k$ acts nontrivially only in $\mathcal{H}_{j}$ and $\mathcal{H}_{k}$ and as the unit operator on the other spaces (the operators $\Omega_{j k}$ retain their form when we replace the $S U(1,1)$ generators by the $S L(2, \mathbb{R})$ generators $\left.\mathbf{e}_{j}, \mathbf{f}_{j}, \mathbf{h}_{j}\right)$.

Solutions of (4.26) for $S U(2)$ were apparently first constructed in [51,52]. The adaptation of these results to the positive discrete series representations of $\operatorname{SL}(2, \mathbb{R})$ reviewed in the appendix works as follows: substituting expressions (A.6) for generators $e_{j}, f_{j}, h_{j}$ in terms of variables $z_{j}$, we see that $\Omega_{j k}$ are differential operators of the second order, and the $\mathrm{KZ}$ equations (4.26) can be realized as the following system of linear partial differential equations for the wave function $\Phi\left(\gamma_{1}, \ldots, \gamma_{N} ; z_{1}, \ldots, z_{N}\right)$ :

$$
\begin{aligned}
\frac{\partial \Phi}{\partial \gamma_{j}}= & -i \hbar \sum_{k \neq j} \frac{1}{\gamma_{j}-\gamma_{k}}\left\{-\left(z_{j}-z_{k}\right)^{2} \frac{\partial^{2} \Phi}{\partial z_{j} \partial z_{k}}+\left(z_{k}-z_{j}\right)\left(s_{j} \frac{\partial \Phi}{\partial z_{k}}-s_{k} \frac{\partial \Phi}{\partial z_{j}}\right)\right. \\
& \left.+\frac{1}{2} s_{j} s_{k} \Phi\right\} .
\end{aligned}
$$

Solutions of these equations corresponding to the positive discrete series correspond to functions which for all $z_{j}$ are holomorphic in the upper half plane. They are described by Theorem 9 .

Theorem 9. The following expression satisfies Eqs. (4.26):

$$
\Phi_{\mathrm{KZ}}=\oint_{\Delta_{1}} d v_{1} \cdots \oint_{\Delta_{M}} d v_{M} W \varphi,
$$

where

$$
\varphi:=e\left(v_{1}\right) \cdots e\left(v_{M}\right)|p\rangle
$$

are the "off-shell" Bethe states introduced in (4.16) and the function $W$ is defined by

$$
\begin{aligned}
W\left(\left\{\gamma_{j}, v_{r}\right\}\right):= & \prod_{1 \leq k<j \leq N}\left(\gamma_{j}-\gamma_{k}\right)^{-i \hbar s_{j} s_{k} / 2} \prod_{1 \leq s<r \leq M}\left(v_{r}-v_{s}\right)^{-2 i \hbar} \\
& \times \prod_{r=1}^{M} \prod_{j=1}^{N}\left(\gamma_{j}-v_{r}\right)^{-i \hbar s_{j}}
\end{aligned}
$$

and is assumed to be single-valued on the cycle $\Delta$ in $\mathbb{C}^{M}$ consisting of a family of $v_{r}$-dependent contours $\Delta_{r}$ in $\mathbb{C}^{M}$ having empty intersection with the hyperplanes $v_{s}=v_{r}$ for $r \neq s$ and the $(\xi, \bar{\xi})$-dependent hypersurfaces $v_{r}=\gamma_{j}$, where $r, s=1, \ldots, M$ and $j=1, \ldots, N$. 
Proof. (See [52,53]). In the remainder we shall use the shorthand notation

$$
\oint_{\Delta} d v \equiv \oint_{\Delta_{1}} d v_{1} \cdots \oint_{\Delta_{M}} d v_{M}
$$

for the multiple contour integral in (4.29). Let us also define

$$
\varphi_{r}:=e\left(v_{1}\right) \cdots e\left(v_{r-1}\right) e\left(v_{r+1}\right) \cdots e\left(v_{M}\right)|p\rangle,
$$

so that

$$
\frac{\partial \varphi_{r}}{\partial v_{r}}=0
$$

since the term $e\left(v_{r}\right)$ is omitted from $\varphi_{r}$. Then by a lengthy but straightforward calculation, taking into account the definiting conditions (4.14), one shows that

$$
H_{j} \varphi=(i \hbar)^{2}\left(\alpha_{j} \varphi-\sum_{r} \frac{\beta_{r}}{\gamma_{j}-v_{r}} e_{j} \varphi_{r}\right),
$$

where

$$
H_{j}=(i \hbar)^{2} \sum_{k \neq j} \frac{\Omega_{j k}}{\gamma_{j}-\gamma_{k}}
$$

are the $\mathrm{KZ}$ Hamiltonians entering (4.26). Here we have defined the functions

$$
\begin{aligned}
\alpha_{j} & =\sum_{i \neq j} \frac{s_{i} s_{j}}{2\left(\gamma_{j}-\gamma_{i}\right)}-\sum_{r} \frac{s_{j}}{v_{r}-\gamma_{j}}, \\
\beta_{r} & =\sum_{s \neq r} \frac{2}{v_{r}-v_{s}}+\sum_{j} \frac{s_{j}}{v_{r}-\gamma_{j}} .
\end{aligned}
$$

By construction, $W$ in (4.31) satisfies

$$
\frac{\partial W}{\partial \gamma_{j}}=-i \hbar \alpha_{j} W, \quad \frac{\partial W}{\partial v_{r}}=-i \hbar \beta_{r} W .
$$

Invoking (4.35) and (4.39), we get

$$
\begin{aligned}
\frac{\partial \Phi_{\mathrm{KZ}}}{\partial \gamma_{j}}+\frac{1}{i \hbar} H_{j} \Phi_{\mathrm{KZ}} & =\oint_{\Delta} d v\left(-\sum_{r} \frac{\partial}{\partial v_{r}}\left\{\frac{e_{j}}{v_{r}-\gamma_{j}}\right\} \varphi_{r}-i \hbar \sum_{r} \frac{\beta_{r} e_{j}}{\gamma_{j}-v_{r}} \varphi_{r}\right) W \\
& =-e_{j} \oint_{\Delta} d v \sum_{r} \frac{\partial}{\partial v_{r}}\left\{\frac{W}{v_{r}-\gamma_{j}} \varphi_{r}\right\}=0,
\end{aligned}
$$

because of (4.34), and there are no boundary contributions as $\Delta$ is closed.

For a discussion of the completeness of the solutions (4.29) in the case of $S U(2)$, see $[52,54]$. The cycles $\Delta$, on which the solutions depend in addition to the parameters 
characterizing the $N$-soliton Hilbert spaces, are generically rather complicated, especially in the limit $\hbar \rightarrow 0$. For $\operatorname{SL}(2, \mathbb{R})$, the space of solutions is infinite dimensional even for fixed soliton number $N$ due to the fact that the underlying Hilbert space $\mathcal{H}^{(N)}$ is also infinite dimensional: unlike for $S U(2)$, the basic formula (4.29) yields nontrivial solutions for arbitrary $M$.

With the solution (4.29) of the KZ equation at hand, we can now proceed to the construction of the full WDW functional $\Phi$ solving (4.25).

Theorem 10. Let $\Phi_{\mathrm{KZ}} \in \mathcal{H}$ be any solution (4.29) of the $\mathrm{KZ}$ equations (4.26). Then the $\mathcal{H}$-valued function $\Phi(\xi, \bar{\xi})$, defined by

$$
\Phi=(\xi-\bar{\xi})^{-\hbar^{2} s_{\infty}\left(s_{\infty}-2\right) / 4} \prod_{j=1}^{N}\left(\frac{\partial \gamma_{j}}{\partial w_{j}}\right)^{-\hbar^{2} s_{j}\left(s_{j}-2\right) / 4} \Phi_{\mathrm{KZ}}\left(\left\{\gamma_{j}\right\}\right)
$$

with

$$
s_{\infty}:=2 M+\sum_{j} s_{j},
$$

satisfies the WDW equation (4.25).

Proof. From (4.2), we find

$$
\operatorname{tr}\left(A_{j} A_{k}\right)=-\hbar^{2} \Omega_{j k} .
$$

Moreover, one can check the following relation between the total Hamiltonians $H^{(\xi)}$, $H^{(\bar{\xi})}$ and the KZ-Hamiltonians $H_{j}$ (3.76) which resembles the classical relation (3.80) between $H^{(\xi)}, H^{(\bar{\xi})}$ and the Schlesinger Hamiltonians. For instance,

$$
\begin{aligned}
H^{(\xi)}= & \sum_{j} H_{j} \gamma_{j \xi}-\frac{1}{\xi-\bar{\xi}} \sum_{j} \operatorname{tr}\left(A_{j}^{2}\right)\left(\frac{1}{2}-\frac{1}{\left(1-\gamma_{j}\right)^{2}}\right) \\
& +\frac{1}{2(\xi-\bar{\xi})} \operatorname{tr}\left(\sum_{j} A_{j}\right)^{2} .
\end{aligned}
$$

The second term on the r.h.s. of (4.42) involves the Casimir operators of the respective $S L(2, \mathbb{R})$ representations and acts on the off-shell Bethe states according to

$$
\operatorname{tr}\left(A_{j}^{2}\right) \varphi=(i \hbar)^{2} \Omega_{j j} \varphi=-\frac{1}{2} \hbar^{2} s_{j}\left(s_{j}-2\right) \varphi .
$$

Writing

$$
-A_{\infty} \equiv \frac{i \hbar}{2}\left(\begin{array}{cc}
\mathbf{h}_{\infty} & 2 \mathbf{e}_{\infty} \\
2 \mathbf{f}_{\infty} & -\mathbf{h}_{\infty}
\end{array}\right)=\frac{i \hbar}{4}\left(\begin{array}{cc}
1 & -i \\
-i & 1
\end{array}\right)\left(\begin{array}{cc}
h_{\infty} & 2 e_{\infty} \\
2 f_{\infty} & -h_{\infty}
\end{array}\right)\left(\begin{array}{cc}
1 & i \\
i & 1
\end{array}\right),
$$

we have

$$
h_{\infty} \varphi \equiv \sum_{j=1}^{N} h_{j} \varphi=\left(2 M+\sum_{j} s_{j}\right) \varphi \equiv s_{\infty} \varphi .
$$


For the last term of (4.42) we then get

$$
\operatorname{tr}\left(\sum_{j} A_{j}\right)^{2} \varphi=(i \hbar)^{2} \sum_{j, k} \Omega_{j k} \varphi=-\hbar^{2}\left(\frac{1}{2} s_{\infty}\left(s_{\infty}-2\right) \varphi+\sum_{j, r} \beta_{r} e_{j} \varphi_{r}\right)
$$

where the functions $\beta_{r}$ and the states $\varphi_{r}$ are defined in (4.38) and (4.33), respectively, and the off-diagonal last term on the r.h.s is due to the action of the operator $e_{j} f_{j}$. The r.h.s. of (4.43) contributes in an obvious way to (4.40). As for (4.45), the second term on the r.h.s., which is not diagonal, does not contribute to $H^{(\xi)} \Phi$ because by Eq. (4.39) and by partial integration (with $\partial \Delta=\emptyset$ ) it gives a term inside the integral (4.29) proportional to

$$
\oint_{\Delta} d v \sum_{j, r} \frac{\partial W}{\partial v_{r}} e_{j} \varphi_{r}=-\oint_{\Delta} d v \sum_{j} e_{j} W \frac{\partial \varphi_{r}}{\partial v_{r}}
$$

which vanishes by (4.34). In this way we arrive at

$$
\operatorname{tr} A_{\infty}^{2} \Phi_{\mathrm{KZ}}=-\frac{1}{2} \hbar^{2} s_{\infty}\left(s_{\infty}-2\right) \Phi_{\mathrm{KZ}}
$$

which is diagonal; therefore, all terms depending explicitly on $(\xi, \bar{\xi})$ can be integrated straightforwardly.

We note the strong similarity of (4.40) with the classical formula (3.30): the prefactor is essentially the same except that we have replaced the classical expressions in the exponent by the eigenvalues of the corresponding operators. The factor involving $(\xi-\bar{\xi})$ can be absorbed into a renormalization of $\Phi$ by an appropriate choice of the function $f(\xi, \bar{\xi})$ in $(4.17)$. The state $\Phi_{\mathrm{KZ}}$ can therefore be regarded as the quantum analog of the classical $\tau$-function ${ }^{5}$.

Formula (4.40) gives the general solution of (4.25) for both $S U(1,1)$ (and therefore $S L(2, \mathbb{R})$ ) and $S U(2)$ for the respective lowest weights $s_{j}$. For $S U(2)$ the theorem was already stated without proof in [2], whereas the noncompact case was not considered there. This leaves us with the task of translating the classical coset conditions of Section 3.3 into the quantum theory. However, we cannot directly generalize the condition $g(\xi, \bar{\xi}) \in S L(2, \mathbb{R}) / S O(2)$ because we do not know the proper definition of the quantum operator corresponding to $g(\xi, \bar{\xi})$. This is why in Section 3.3 we explored different ways to formulate the coset constraint. The similarity between $\tau$ and $\Phi_{\mathrm{KZ}}$ suggests the necessary conditions,

$$
\left(\sum_{j=1}^{N} A_{j}\right) \Phi_{\mathrm{KZ}}=0
$$

\footnotetext{
${ }^{5}$ Alternatively, one could identify the quantum $\tau$-function with the corresponding evolution operator which is a "matrix" whose columns constitute an orthonormal basis of states $\Phi_{\mathrm{KZ}}$ in the space of solutions of KZ equations.
} 
and

$$
\Phi_{\mathrm{KZ}}\left(\frac{1}{\gamma_{1}}, \ldots, \frac{1}{\gamma_{N}}\right)=\Phi_{\mathrm{KZ}}\left(\gamma_{1}, \ldots, \gamma_{N}\right) .
$$

These imply the following restrictions on the parameters of $\Phi_{\mathrm{KZ}}$, and hence on the full WDW wave functional $\Phi$ by Theorem 10 .

Theorem 11. The solution $\Phi_{\mathrm{KZ}}$ in (4.29) of the $\mathrm{KZ}$ equations satisfies the symmetry conditions (4.47) and (4.48) if $N=2 n, \gamma_{j+n}=\gamma_{j}^{-1}$ and $s_{j+n}=s_{j}(j=1, \ldots, n)$,

$$
s_{\infty} \equiv 2 M+\sum_{j=1}^{N} s_{j}=0,
$$

and the cycle $\Delta$ in (4.29) is invariant with respect to the continuous deformation of $\gamma_{j}$ into $\gamma_{j}^{-1}$.

Unfortunately, there is a simple argument showing that (4.49) cannot be satisfied for the noncompact theory with the discrete representations of $S U(1,1)$ or $S L(2, \mathbb{R})$, unlike for the compact group $S U(2)$ (for which $s_{j} \leq 0$ ). Namely, for the noncompact groups we have $s_{j}>0$ and condition (4.49) can never be met; switching to the negative discrete series, for which $s_{j}<0$, does not help because $M$ becomes negative due to the interchange of $e_{j}$ and $f_{j}$. Therefore it appears that the discrete representations of $S U(1,1)$ are unsuitable for the task at hand, at least as long as we do not make simultaneous use of the positive and negative series. This conclusion is confirmed by an analysis of the sign of the Casimir operator (cf. (4.52) below) which shows explicitly that the known classical solutions are associated with the principal series representations of $S L(2, \mathbb{R})$ or $S L(2, \mathbb{C})$. Moreover, let us repeat that for $\gamma_{j}=-\overline{\gamma_{k}}$, when $\mathcal{H}_{j}$ carries a unitary representation of $S L(2, \mathbb{C})$, the discrete series are altogether absent, so the consideration of the principal series cannot be avoided in any case. Finally, a complete treatment of the quantum coset constraints will require the implementation of (3.49) at the quantum level.

As an aside we observe that the "ultra quantum limit" $\hbar \rightarrow \infty$ corresponds to the semi-classical limit of the WZNW model (and vice versa). Heuristically, this limit is dominated by the stationary points of the integral which are determined by the equations $\beta_{j}=0$, or, equivalently,

$$
\sum_{s \neq r} \frac{2}{v_{r}-v_{s}}+\sum_{j} \frac{s_{j}}{v_{r}-\gamma_{j}}=0 .
$$

These are nothing but the so-called Bethe equations $[13,14]$ which diagonalize the "matter Hamiltonians" $H_{j}$ on the Bethe states (4.16), except that the Bethe parameters $v_{r}$ here depend on the coordinates because $\gamma_{j}=\gamma_{j}(\xi, \bar{\xi})$. Consequently, in the limit $\hbar \rightarrow \infty$, the quantum states become more and more sharply peaked about the "on-shell" Bethe states. 
To study the classical limit of the quantum states we have to consider the expectation values of the various observables and their $\hbar \rightarrow 0$ limits. Although this is a difficult problem, it is at least clear that in order to end up with a nontrivial classical solution the expectation values $\left\langle\operatorname{tr} A_{j}^{2}\right\rangle$ must stay finite in the limit $\hbar \rightarrow 0$. This can be achieved by letting $\hbar \rightarrow 0$ and $s_{j} \rightarrow \infty$ in such a fashion that the product $\hbar s_{j}$ remains finite, i.e.,

$$
\hbar s_{j} \rightarrow s_{j}^{(0)} \neq 0 \text { as } \hbar \rightarrow 0,
$$

such that for discrete representations,

$$
\lim _{\hbar \rightarrow 0}\left\langle\operatorname{tr} A_{j}^{2}\right\rangle=-\lim _{\hbar \rightarrow 0} \hbar^{2}\left\langle\frac{1}{2} h_{j}^{2}+e_{j} f_{j}+f_{j} e_{j}\right\rangle=-\frac{1}{2}\left(s_{j}^{(0)}\right)^{2}<0,
$$

since the operator in parentheses is just the Casimir operator. On the other hand, $\operatorname{tr} A_{j}^{2}$ is positive for all known classical solutions, and this indicates again that we must eventually consider solutions based on the principal series representation for which the expectation value $\left\langle\operatorname{tr} A_{j}^{2}\right\rangle$ is positive.

\section{Stringy aspects}

To conclude we would like to emphasize the intriguing analogies of our results with certain aspects of string theory and the tantalizing hints of a new type of theory which could emerge upon a "stringy" reinterpretation of (a supersymmetric version of) dimensionally reduced quantum gravity along the lines already suggested in [56]. The similarity of the dilaton field $\rho$ and the logarithm of the conformal factor with the longitudinal target space coordinates $X^{+}$and $X^{-}$, respectively, was already pointed out in [36]. Furthermore, the choice of Weyl canonical coordinates in (2.11) corresponds to the lightcone gauge fixing condition $X^{+}=\tau$ in string theory; in [21] it was shown that this analogy remains valid for higher genus worldsheets if one identifies $\rho$ with the globally defined (lightcone) time coordinate introduced by Mandelstam to describe string scattering in the lightcone gauge. The fact that through this choice of gauge the field variables $2 k_{\xi}$ and $2 k_{\bar{\xi}}$ become canonically conjugate to the worldsheet coordinates is also in accord with this interpretation. The transmutation of the conformal factor into a longitudinal target space degree of freedom has also been proposed in Liouville theory (i.e., subcritical string theory) [57]; however, the Lagrangians considered for this purpose apparently do not arise from a dimensional reduction of Einstein's theory.

We have also remarked that the WDW equations (4.24) should be considered on a par with the Virasoro constraints of string theory as they state nothing but the vanishing of the off-diagonal components $T_{\xi \xi}$ and $T_{\bar{\xi} \bar{\xi}}$ of the full energy-momentum tensor on the physical states, where the full energy-momentum tensor is defined to include the gravitational contribution $\partial_{\xi} k \equiv 2 i \partial_{\xi} k \partial_{\xi} \rho$ (or its Hermitean conjugate), again in analogy with the Liouville theory. Thus instead of regarding the expressions (4.40) as solutions of $2 \mathrm{~d}$ matter coupled quantum gravity, we could alternatively interpret them as physical states in some higher-dimensional target space. The operators $e(\gamma)$ and $f(\gamma)$ would 
play the role of transverse creation and annihilation operators, respectively. We note that the relevant "oscillators" are not the Fourier coefficients of the string target space coordinates with respect to the left and right moving worldsheet coordinates $\xi$ and $\bar{\xi}$, but rather based on an expansion with respect to the spectral parameter $\gamma$ which appears to be the truly fundamental variable. Our hypothetical string would thus be neither closed nor open, but "unidexterous". The similarity between the contour integrals over products of "oscillators" appearing in (4.40) and the corresponding expressions giving physical string states in terms of DDF operators is noteworthy (in fact, our expressions are almost identical with the integrals for correlators of the $c<1$ minimal conformal models [51]).

Of course, we would not expect the new "string" to be automatically consistent; instead, consistent models should satisfy further constraints such as absence of anomalies (which is the criterion singling out the critical string theories). As is well known, the crucial consistency test in the lightcone gauge is the closure of the Lorentz algebra (see, e.g., [58]), and in addition to defining the target space we will have to look for the analog of the Lorentz algebra. The obvious candidate for the transverse subgroup for the model investigated here is the $S O(2)$ subgroup of the Ehlers group $S L(2, \mathbb{R})$ generated by $A_{\infty}$ (see (3.14)), but the extension to a full Lorentz group remains to be found.

One of the outstanding problems of midi-superspace quantum gravity is to find a symplectic (canonical) realization of the Geroch group [34]. Classically this is simply the group of "dressing transformations" which add extra regular singularities to $\Psi(\gamma)$ with special monodromy data. Here we have investigated the isomonodromic sectors separately, where only representations of $S L(2, \mathbb{R})$ appear, but the general case should involve the affine extension $S \widehat{L(2, \mathbb{R})}$. The appearance of the involution $\gamma \rightarrow \gamma^{-1}$ in (3.50) and (4.48) moreover suggests that in a fully covariant formulation the physical states should be invariant under the "maximal compact" subgroup $S O(2)^{\infty}$ already encountered in $[35,33,36]$. While there are indications from flat space models that such transformations cannot be realized within the conventional canonical approach [59], the situation may be different for our new formulation. The quantum Geroch group would relate the quantum states to one another in the same way that the ordinary Geroch group relates classical solutions; it would mix the isomonodromic sectors and change the soliton number $N$. The monodromy operators (3.17) are the natural candidates for conserved quantum nonlocal charges. The work of [49] on the quantum group structure of the monodromy algebra of the $\mathrm{KZ}$ equations suggests that the Geroch group could become a true quantum group in the technical sense of the word (see also [50]). In the string context the quantum Geroch group would be interpreted as a spectrum generating symmetry with the physical states belonging to unitary representations of the relevant noncompact (quantum) group.

The introduction of interactions between physical string states would amount to a "third quantization" from the $2 \mathrm{~d}$ worldsheet point of view, such that scattering processes involving different physical states would correspond to the interaction of different $2 \mathrm{~d}$ "universes". However, just as the construction of a proper string field theory requires more than the Virasoro constraints, such an interpretation of our model would involve 
essentially new elements beyond the WDW equations (4.24). In particular, it would necessitate repeating the analysis of this paper for higher genus worldsheets. Readers may appreciate the resemblance of these ideas with recent attempts to understand the possible loss of quantum coherence and the emergence of baby universes in quantum gravity on the basis of certain $2 \mathrm{~d}$ models [60]; however, our intentions here really go in the opposite direction as we wish to build a new kind of string theory from these models rather than to treat string theory as an ancillary model to understand features of $2 \mathrm{~d}$ quantum gravity.

Finally, the manifest split into left and right moving sectors put in evidence by the automatic compatibility of (3.22) and the mutual commutativity of the Hamiltonians (3.54) as well as the canonical constraints (3.60) is strongly reminiscent of holomorphic factorization in string theory. It suggests that our formulation is the natural starting point for studying the reduction to one dimension. In analogy with string theory, which can be regarded as a $2 d$ field theory composed of two one-dimensional (chiral) halves, such a reduction would not really be a dimensional reduction to one dimension. One might even argue that the $2 \mathrm{~d}$ theory is already "one-dimensional" in that the $(\xi, \bar{\xi})$-dependence essentially enters only via the (analytic) dependence on one complex variable $\gamma$. In contrast to a naive dimensional reduction of the original theory, which would just leave us with trivial plane waves, the rich structure of stationary axisymmetric or colliding plane wave quantum gravity would be entirely preserved in this scheme. This also indicates that the spectrum of the new theory would contain many more excitations than the ordinary string because the structure of unitary representations of $\operatorname{SL}(2, \mathbb{R})$ and other noncompact groups (see [61]) is considerably more intricate than the "linear" harmonic oscillator spectra of string theory, leaving room for myriad solitonic excitations at the quantum level.

\section{Acknowledgements}

We would like to thank A. Ashtekar, H. Babujan, M. Günaydin, B. Julia, A. Kitaev, J. Lewandowski, H.-J. Matschull, A. Mironov, A. Morosov, H. Samtleben, V. Schomerus, M. Semenov-Tian-Shansky, F. Smirnov and J. Teschner, who have at various stages contributed insightful comments to the present work. D.K. acknowledges the support of the A.v. Humboldt Stiftung and Deutsche Forschungsgemeinschaft under contract No. Ni 290/5-1.

\section{Appendix A. Unitary representations of $S L(2, \mathbb{R})$}

For the convenience of the reader we here summarize some pertinent results about unitary representations of $S L(2, \mathbb{R})$; see [47] for details and further information. All representation spaces $\mathcal{H}^{(s)}$ can be realized as Hilbert spaces of functions $\varphi(z)$ of one (complex or real) variable $z$. The action of the group element $G \in S L(2, \mathbb{R})$, 


$$
G=\left(\begin{array}{ll}
a & b \\
c & d
\end{array}\right)
$$

(where $a d-b c=1$ ), on any such function is given by

$$
T_{s}(G) \varphi(z)=\varphi\left(\frac{a z+b}{c z+d}\right)(c z+d)^{-s},
$$

if $s$ is integer; otherwise (i.e., for continuous representations) we have $|c z+d|^{-s}$ instead of $(c z+d)^{-s}$ on the r.h.s. of this formula. For all representations, the Chevalley generators with commutation relations

$$
[\mathbf{h}, \mathbf{e}]=2 \mathbf{e}, \quad[\mathbf{h}, \mathbf{f}]=-2 \mathbf{f}, \quad[\mathbf{e}, \mathbf{f}]=\mathbf{h},
$$

are represented on $\mathcal{H}^{(s)}$ by the differential operators

$$
T_{s}(\mathbf{e})=z^{2} \frac{d}{d z}+s z, \quad T_{s}(\mathbf{f})=-\frac{d}{d z}, \quad T_{s}(\mathbf{h})=2 z \frac{d}{d z}+s,
$$

where the parameter $s$ must satisfy the constraints given below. These operators are anti-Hermitean with respect to the scalar products given below. The Casimir operator $T_{s}\left(\frac{1}{2} \mathbf{h}^{2}+\mathbf{e f}+\mathbf{f e}\right)$ is always diagonal; by direct computation one easily verifies that its eigenvalue is $\frac{1}{2} s(s-2)$.

All unitary irreducible representations of $S L(2, \mathbb{R})$ (with the exception of the socalled limit of the discrete series corresponding to $s=1$ ) are contained in the following list.

- For the principal series the functions $\varphi(z)$ live on the real line, i.e., $z \in \mathbb{R}$, and the scalar product is the ordinary $L^{2}(\mathbf{R})$ product (which is independent of $s$ ),

$$
(\psi, \varphi)=\int_{\mathbb{R}} \overline{\psi(z)} \varphi(z) d z
$$

The allowed values for $s$ are $s=1+i q, q \in \mathbb{R}$, and the spectrum of the operators $T_{s}[\mathbf{e}], T_{s}[\mathbf{f}]$ and $T_{s}[\mathbf{h}]$ is continuous for all such $s$.

- For the supplementary series, the functions $\varphi(z)$ are again defined on the real axis, but the scalar product now depends on $s$ and is given by

$$
(\psi, \varphi)_{s}=\int_{\mathbf{R}^{2}} \overline{\psi\left(z_{1}\right)} \varphi\left(z_{2}\right)\left|z_{1}-z_{2}\right|^{s-2} d z_{1} d z_{2},
$$

with $s \in \mathbb{R}$ and $0<s<2$ (the latter restriction follows from requiring $(\varphi, \varphi)_{s}$ to be positive for nonzero $\varphi$ ). The spectrum of the operators $T_{s}[\mathbf{e}], T_{s}[\mathbf{f}], T_{s}[\mathbf{h}]$ is again continuous.

- The positive discrete series representations consist of the functions holomorphic in the upper half plane normalizable with respect to the scalar product

$$
(\psi, \varphi)_{s}:=\int_{\operatorname{Im} z>0} \overline{\psi(z)} \varphi(z)|\operatorname{Im} z|^{s-2} d z d \bar{z} .
$$


In order to ensure single-valuedness of the functions $\varphi(z)$, only discrete values of $s$ are admitted; furthermore, convergence of the integral at $\operatorname{Im} z=0$ requires $s \geq 2$, so we have $s=2,3, \ldots$.

To construct an explicit basis of functions for the discrete series, the $S U(1,1)$ basis is more useful; it is realized by the operators

$$
\begin{aligned}
& T_{s}(e)=\frac{1}{2} T_{s}(-i \mathbf{h}+\mathbf{e}+\mathbf{f})=\frac{1}{2}(z-i)^{2} \frac{d}{d z}+\frac{1}{2} s(z-i), \\
& T_{s}(f)=\frac{1}{2} T_{s}(i \mathbf{h}+\mathbf{e}+\mathbf{f})=\frac{1}{2}(z+i)^{2} \frac{d}{d z}+\frac{1}{2} s(z+i), \\
& T_{s}(h)=T_{s}(i(\mathbf{f}-\mathbf{e}))=-i(z-i)(z+i) \frac{d}{d z}-i s z
\end{aligned}
$$

We can explicitly check that

$$
\begin{aligned}
& {\left[T_{s}(h), T_{s}(e)\right]=2 T_{s}(e), \quad\left[T_{s}(h), T_{s}(f)\right]=-2 T_{s}(f),} \\
& {\left[T_{s}(e), T_{s}(f)\right]=T_{s}(h),}
\end{aligned}
$$

as well as

$$
T_{s}(h)=T_{s}(h)^{\dagger}, \quad T_{s}(e)=-T_{s}(f)^{\dagger},
$$

with respect to the scalar product (A.5). The representation space $\mathcal{H}_{+}^{(s)}$ of the positive discrete series is spanned by the following functions holomorphic in the upper half-plane:

$$
\varphi_{s}^{s+k}(z)=(z-i)^{k}(z+i)^{-s-k}, \quad k=0,1,2, \ldots
$$

The generators $T_{s}(e), T_{s}(f)$ and $T_{s}(h)$ act on these functions as follows:

$$
\begin{aligned}
& T_{s}(h) \varphi_{s}^{s+k}=(s+2 k) \varphi_{s}^{s+k}, \quad T_{s}(e) \varphi_{s}^{s+k}=i(s+k) \varphi_{s}^{s+k+1}, \\
& T_{s}(f) \varphi_{s}^{s+k}=i k \varphi_{s}^{s+k-1} .
\end{aligned}
$$

The lowest weight state corresponds to the function $\varphi_{s}^{s}$ which is annihilated by $T_{s}(f)$ and from which all other functions can be generated by repeated application of the raising operator $T_{s}(e)$; also the operator $T_{s}(h)$ is diagonal in this basis with eigenvalues $s, s+2, s+4, \ldots$.

\section{References}

[1] D. Korotkin and H. Nicolai, Phys. Rev. Lett. 74 (1995) 1272.

[2] D. Korotkin and H. Nicolai, Phys. Lett. B 356 (1995) 211.

[3] S.W. Hawking, in: Relativity, Groups and Topology II, eds. B. DeWitt and R. Stora (North-Holland, Amsterdam, 1984).

[4] C. Isham, in: Recent Aspects of Quantum Fields, eds. H. Mitter and H. Gausterer (Springer, Berlin, 1991).

[5] A. Ashtekar, in: Gravitation and Quantizations, eds. B. Julia and J. Zinn Justin (North-Holland, Amsterdam, 1995).

[6] E. Witten, Nucl. Phys. B 311 (1988) 46. 
[7] S. Carlip, Lectures on (2+1) dimensional gravity, preprint gr-qc-9503024; H.J. Matschull, J. Class. and Quant. Grav. 12 (1995) 2621.

[8] B. de Wit, H.J. Matschull and H. Nicolai, Phys. Lett. B 318 (1993) 115.

[9] H. Kastrup and T. Thiemann, Nucl. Phys. B 425 (1994) 665.

[10] S. Elitzur, A. Forge and E. Rabinovici, Nucl. Phys. B 274 (1986) 60;

R. Graham and A. Csordás, Phys. Rev. Lett. 74 (1995) 4129.

[11] K. Kuchar, Phys. Rev. D 4 (1971) 955.

[12] A. Ashtekar and M. Pierri, to appear.

[13] L. Faddeev and L. Takhtajan, Hamiltonian Methods in the Theory of Solitons (Springer, Berlin, 1986).

[14] V. Korepin, M. Bogoliubov and A. Izergin, Quantum Inverse Scattering Method and Correlation Functions (Cambridge Univ. Press, Cambridge, 1993).

[15] V. Knizhnik and A. Zamolodchikov, Nucl. Phys. B 247 (1984) 83.

[16] J. Wess and B. Zumino, Nucl. Phys. B (Proc. Suppl.) 18B (1991) 302;

S. Doplicher, K. Fredenhagen and J.E. Roberts, Phys. Lett. B 331 (1994) 39.

[17] S.W. Hawking, Phys. Rev. D 14 (1976) 2460;

G. 't Hooft, Nucl. Phys. B 335 (1990) 138;

for recent reviews with many further references, see T. Banks, Lectures on black holes and information loss, preprint RU-94-91;

F. Englert, Operator weak values and black hole complementarity, preprint ULB-TH 03/95.

[18] F. Ernst, Phys. Rev. 167 (1968) 1175.

[19] D. Kramer, H. Stephani, E. Herlt and M. MacCallum, Exact Solutions of Einstein's Field Equations (Cambridge Univ. Press, Cambridge, 1980).

[20] D. Kramer and G. Neugebauer, Commun. Math. Phys. 10 (1968) 132.

[21] D. Korotkin and H. Nicolai, Nucl. Phys. B 429 (1994) 229.

[22] T, Thiemann, Reality conditions inducing transforms for quantum gauge field theory and quantum gravity, preprint gr-qc/9511057;

A. Ashtekar, A generalized Wick transform for gravity, preprint gr-qc/9511083.

[23] D. Maison, Phys. Rev. Lett. 41 (1978) 521.

[24] V. Belinskii and V. Zakharov, Sov. Phys. JETP 48 (1978) 985.

[25] G. Neugebauer and D. Kramer, J. Math. Phys. 32 (1991) 2727.

[26] M. Jimbo, T. Miwa, T. Mori and Y. Sato, Physica D 1 (1980) 80;

M. Jimbo, T. Miwa and K. Ueno, Physica D 2 (1981) 306.

[27] H. Nicolai, Nucl. Phys. B 414 (1994) 299.

[28] D. Korotkin, Theor. Math. Phys. 77 (1989) 1018;

D. Korotkin and V. Matveev, St. Petersburg Math. J. 1 (1990) 379;

D. Korotkin, Class. Quant. Grav. 100 (1993) 2587; Commun. Math. Phys. 137 (1991) 383; Zapiski Nauch. Sem. LOMI (1993).

[29] D. Korotkin, On some integrable cases in surface theory, Berlin preprint SFB 288 No. 116, TU Berlin (1994).

[30] S. Persides and B. Xantopoulos, J. Math. Phys. 29 (1988) 674;

A. Kitaev, Zap. Nauch. Sem. LOMI 181 (1990) 65.

[31] R.C. Myers, Phys. Rev. D 35 (1987) 455.

[32] A.R. Its, Math USSR Isvestija 26 (1985) 497.

[33] P. Breitenlohner and D. Maison, Ann. Inst. Poincaré 46 (1987) 215.

[34] R. Geroch, J. Math. Phys. 12 (1971) 918.

[35] B. Julia, in: Unified Theories and Beyond, Proc. 5th Johns Hopkins Workshop on Current Problems in Particle Theory, Johns Hopkins University, Baltimore (Johns Hopkins University, Baltimore, 1980).

[36] H. Nicolai, in: Recent Aspects of Quantum Fields, eds. H. Mitter and H. Gausterer (Springer, Berlin, 1991).

[37] I. Kanatchikov, On the canonical structure of the De Donder-Weyl covariant Hamiltonian formulation of field theory, preprint hep-th/9312162;

N.P. Landsmann, Against the Wheeler-DeWitt equation, preprint gr-qc/9510033;

H.J. Matschull, Causal structure and diffeomorphisms in Ashtekar's gravity, preprint gr-qc/9511034.

[38] P.A.M. Dirac, Lectures on Quantum Mechanics (Academic Press, New York, 1967).

[39] D. Korotkin, $H$. Nicolai and $H$. Samtleben, On 2D quantum gravity coupled to a $\sigma$-model, Nucl. Phys. B (Proc. Suppl.) 49 (1996) pp. 1-10, “Theory of Elementary Particles”, eds. D. Lüst, G. Weigt. 
[40] V. Fock and A. Rosly, Poisson structure on the moduli space of flat connections on Riemann surfaces and $r$-matrix, preprint ITEP 72-92, Moscow (June 1992).

[41] D. Korotkin and H. Samtleben, On the quantization of isomonodromic deformations on the torus, preprint hep-th/9511087.

[42] H. de Vega, H. Eichenherr and J.M. Maillet, Commun. Math. Phys. 92 (1984) 507; J.M. Maillet, Nucl. Phys. B 269 (1986) 54.

[43] L. Faddeev and N. Reshetikhin, Ann. Phys. 167 (1986) 227.

[44] A. Duncan, M. Niedermaier and H. Nicolai, Z. Phys. C 46 (1990) 147.

[45] L. Schlesinger, J. Reine u. Angew. Math. 141 (1912) 76.

[46] H.D. Zeh, The Physical Basis of the Direction of Time (Springer, Berlin, 1992);

C.J. Isham, in: Canonical Relativity: Classical and Quantum, eds. J. Ehlers and H. Friedrich (Springer, Berlin, 1994).

[47] A. Barut and C. Fronsdal, Proc. Royal Soc. A 287 (1965) 532;

I.M. Gelfand, M.I. Graev and N. Ya. Vilenkin, Generalized Functions, Vol. 5 (Academic Press, New York, London, 1966);

S. Lang, $S L_{2}(R)$, Graduate Texts in Mathematics, Vol. 105 (Springer, Berlin, 1985);

W. Schmidt, in: Representation Theory of Lie Groups, ed. M. Atiyah (Cambridge Univ. Press, Cambridge, 1979).

[48] A. Ashtekar and J. Lewandowski, J. Math. Phys. 36 (1995) 2170; Differential geometry on the space of connections via graphs and projective limits, preprint hep-th/9412073.

[49] V. Drinfeld, Quasi-Hopf algebras, Leningrad Math. J. 1 (1990).

[50] A. Alekseev and V. Schomerus, Representation theory of Chern-Simons observables, preprint qalg/95003016.

[51] V. Dotsenko and V. Fateev, Nucl. Phys. B 240 (1984) 312.

[52] V.V. Schechtman and A.N. Varchenko, Lett. Math. Phys. 20 (1990) 279.

[53] H.M. Babujian, J. Phys. A 26 (1993) 6981;

H. Babujan and R. Flume, Off-Shell Bethe ansatz equations for Gaudin magnets and solutions of Knizhnik-Zamolodchikov equations, Bonn preprint HE-93-90.

[54] A.N. Varchenko, Multidimensional hypergeometric functions and representation theory of Lie algebras and quantum groups, Adv. Studies in Math. Phys. (World Scientific, Singapore, 1995).

[55] A.M. Polyakov, Mod. Phys. Lett. A 2 (1987) 893.

[56] H. Nicolai, Phys. Lett. B 194 (1987) 402.

[57] S. Das and S. Wadia, Mod. Phys. Lett. A 4 (1989) 1033;

J. Polchinski, Nucl. Phys. B 324 (1989) 123.

[58] M.B. Green, J.H. Schwarz and E. Witten, Superstring Theory (Cambridge Univ. Press, Cambridge, 1986).

[59] M. Lüscher and K. Pohlmeyer, Nucl. Phys. B 137 (1978) 46;

M.C. Davies, P.J. Houston, J.M. Leinaas and A. Macfarlane, Phys. Lett. B 119 (1982) 187.

[60] S.B. Giddings and A. Strominger, Nucl. Phys. 307 (1988) 854;

A. Lyons and S.W. Hawking, Phys. Rev. D 44 (1991) 3802;

V.A. Rubakov, Modelling macroscopic and baby universes by fundamental strings, preprint hepth/9505159.

[61] M. Günaydin and C. Saçlioğlu, Commun. Math. Phys. 87 (1982) 159. 